\title{
HOLOMORPHIC SELF-MAPS OF THE DISK INTERTWINING TWO LINEAR FRACTIONAL MAPS
}

\author{
MANUEL D. CONTRERAS, SANTIAGO DÍAZ-MADRIGAL, MARÍA J. MARTÍN, \\ AND DRAGAN VUKOTIĆ
}

\begin{abstract}
We characterize (in almost all cases) the holomorphic selfmaps of the unit disk that intertwine two given linear fractional selfmaps of the disk. The proofs are based on iteration and a careful analysis of the Denjoy-Wolff points. In particular, we characterize the maps that commute with a given linear fractional map (in the cases that are not already known) and, as an application, determine all "roots" of such maps in the sense of iteration (if any). This yields as a byproduct a short proof of a recent theorem on the embedding of a linear fractional transformation into a semigroup of holomorphic self-maps of the disk.
\end{abstract}

\section{Contents}

Introduction 2

1. Some background 4

1.1. Two simple criteria for an LFT to map the disk into itself 4

1.2. Angular limits and derivatives 5

1.3. Iteration and Denjoy-Wolff points 5

1.4. Standard simplifications by conjugation 6

2. Conformality of solutions and compatibility of dynamic types 7

2.1. Conformality of solutions $\quad 7$

2.2. Compatibility of dynamic types of the intertwining LFT's 9

3. A rigidity principle and further results on the intertwining equation

3.1. A rigidity principle for intertwining $\quad 16$

3.2. The case when $\varphi$ is an elliptic automorphism 18

3.3. The case when $\varphi$ is a parabolic automorphism 20

3.4. The intertwining equation and the sets of fixed points 21

Date: 06 August, 2010.

2000 Mathematics Subject Classification. Primary: 30D05. Secondary: 37F10.

Key words and phrases. Intertwining, commutation, linear fractional map, disk automorphism, angular derivative, iteration, Denjoy-Wolff point.

This work is supported by coordinated research grants from MICINN, Spain: the first two authors are supported by MTM2009-14694-C02-02 co-financed by FEDER (European Regional Development Fund) and the remaining two authors by MTM2009-14694-C0201. All authors are also partially supported by the Thematic Network (Red Temática) MTM2008-02829-E, Acciones Especiales, MICINN. The first two and the fourth author are also partially sponsored by the ESF Network HCAA. 
4. Some applications 24

4.1. Commutation 24

4.2. Roots 26

4.3. A remark on the Koenigs embedding problem for semigroups 29

References

\section{INTRODUCTION}

In what follows, writing $f \in \mathcal{H}(\mathbb{D}, \mathbb{D})$ or saying that $f$ is a self-map of the unit disk $\mathbb{D}$ will mean that $f$ is analytic in $\mathbb{D}$ and $f(\mathbb{D}) \subset \mathbb{D}$. We will be particularly interested in linear fractional transformations: $\varphi(z)=$ $(a z+b) /(c z+d), a d-b c \neq 0$, from now on, often abbreviated as LFT's. The main purpose of this paper is to study the self-maps $f$ of $\mathbb{D}$ that intertwine two prescribed linear fractional self-maps $\varphi$ and $\psi$ of the disk: $f \circ \varphi=\psi \circ f$.

Our study is inspired by the special case when $\varphi=\psi$; that is, $f \circ \varphi=\varphi \circ f$. Related questions have a long history which started with the pioneering works by Shields [24] and Behan [3] on families of commuting self-maps of the disk and continued with Cowen's articles $[12,13]$. A considerable amount of work has been done by Italian authors: see [4], [7], [9], or [28], to mention only a few papers in the context of one complex variable. A relationship with the so-called polymorphic functions [19] should also be mentioned. Several partial results regarding the commutation with LFT's can be found in the recent paper [15].

Two classical examples of intertwining relations are Schroeder's equation and Abel's equation from complex dynamics, where one of the intertwined maps is an LFT [27], [18], [10]. They are also important in the theory of composition operators $[14,22,23]$ where they are used to analyze, for example, the compactness and the spectrum.

Our aim is to show that in most situations where $\varphi$ and $\psi$ are both LFT's, the intertwining equation $f \circ \varphi=\psi \circ f$ forces $f$ to be an LFT as well. Such rigidity principles are frequent in the studies involving the use of different generalizations of the Schwarz Lemma or its boundary versions [1], [6], [9]. One of the key points in answering the question on intertwining is precisely a careful analysis of the Denjoy-Wolff points of the maps $\varphi$ and $\psi$ and the behavior of $f$ at these points.

It should be noted, however, that there are quite a few exceptions to the rigidity principle in certain special situations. In order to make this clear, appropriate examples are provided wherever needed. We establish that the intertwining equation can only hold for certain combinations of types of $\varphi$ and $\psi$. Also, a conformality property of $f$ at the Denjoy-Wolff point of $\varphi$ is often relevant in this context. Even proving these initial properties seems to require a considerable amount of work.

We now indicate how the paper is organized and comment on some of our main results. In order to make the article as self-contained as possible, the 
essential background will be reviewed where needed but most preliminary facts are listed in Section 1.

Following the standard classification of the non-identity self-maps of $\mathbb{D}$ into three cases: elliptic, parabolic, and hyperbolic, depending on the location and properties of the Denjoy-Wolff points, in Section 2 we analyze the compatibility of dynamic types of $\varphi$ and $\psi$ as a prerequisite for satisfying the intertwining equation $f \circ \varphi=\psi \circ f$. That is, given two linear fractional self-maps $\varphi$ and $\psi$ of the disk of prescribed types, where $\varphi \neq i d_{\mathbb{D}}$ and $\tau$ is the Denjoy-Wolff point of $\varphi$, the question is: does there exist a non-constant analytic function $f: \mathbb{D} \rightarrow \mathbb{D}$ conformal at $\tau$ and satisfying $f \circ \varphi=\psi \circ f$ ? The main results of Section 2 are summarized in the table below.

Table 1

\begin{tabular}{|c|c|c|c|c|c|c|c|c|}
\hline \multirow{2}{*}{\multicolumn{2}{|c|}{$\varphi \psi$}} & \multirow{2}{*}{$\operatorname{id}_{\mathbb{D}}$} & \multicolumn{2}{|c|}{$\overline{\text { Elliptic }}$} & \multicolumn{2}{|c|}{ Hyperbolic } & \multicolumn{2}{|c|}{ "Parabolic } \\
\hline & & & Aut. & $\mathrm{N}-\mathrm{A}$ & Aut. & $\mathrm{N}-\mathrm{A}$ & Aut. & $\mathrm{N}-\mathrm{A}$ \\
\hline \multirow[t]{2}{*}{ Ell. } & $\overrightarrow{\text { Aut. }}$ & $\overline{c \beta}$ & $\overline{\overline{c \beta}}$ & $\overline{\mathrm{No}}$ & $\overline{\mathrm{No}}$ & $\overline{\mathrm{No}}$ & $\overline{\mathrm{N}} \mathrm{No}$ & $\overline{\overline{\mathrm{No}}}$ \\
\hline & $\mathrm{N}-\mathrm{A}$ & No & No & 8 & $\mathrm{No}$ & No & No & No \\
\hline \multirow[t]{2}{*}{ "Hyp. } & Aut. & $\overline{\mathrm{No}}$ & $\overline{\mathrm{No}}$ & $\overline{83}$ & $\overline{\overline{\alpha \beta}}$ & $\overline{\mathrm{No}}$ & $\overline{\mathrm{No}}$ & $\overline{\mathrm{No}}$ \\
\hline & $\mathrm{N}-\mathrm{A}$ & No & No & s & क, & \& & $\mathrm{No}$ & $\mathrm{No}$ \\
\hline \multirow[t]{2}{*}{ Par. } & $\overline{\text { Aut. }}$ & $\overline{c, 3}$ & $\overline{\mathrm{No}}$ & $\overline{\overline{\mathrm{No}}}$ & $\overline{\mathrm{No}}$ & $\overline{\mathrm{No}}$ & $\overline{\bar{\beta}}$ & $\overline{\overline{\mathrm{No}}}$ \\
\hline & $\mathrm{N}-\mathrm{A}$ & No & $\mathrm{No}$ & $\mathrm{No}$ & No & No & No & d. \\
\hline
\end{tabular}

The answer No in each case means that no such $f$ can exist regardless of the choice of $\varphi$ and $\psi$ of the types required. The symbol \& indicates that for some LFT's $\varphi$ and $\psi$ of the type specified one can find a function $f$ satisfying the conditions specified above. The abbreviations Aut and N-A mean automorphic and non-automorphic respectively.

Once we know the cases when such an $f$ exists, it is natural to ask whether it is necessarily a linear fractional map. In Section 3 we consider in detail all possible cases of intertwining and obtain several rigidity principles of this type. A separate and lengthy analysis in each different case is required in some theorems. The results are given at a glance in the following table.

Table 2

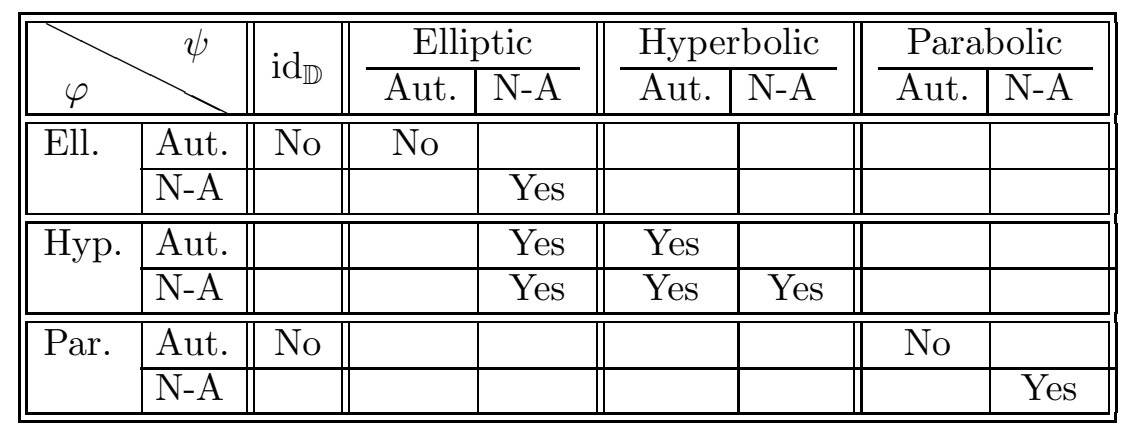

(Naturally, blank spaces correspond to the cases ruled out by Table 1.) 
In Section 4 we give several applications of the results obtained and the methods employed in Section 2 and Section 3. In particular, we solve completely the following problem: when does a self-map $f$ of the disk commute with a linear fractional self-map $\varphi$ of the disk? It should be noted that even in this special case a direct application of earlier, seemingly more general, results is not sufficient and additional discussions are required in order to deduce the desired conclusions.

The paper ends with a new approach on what is sometimes called the Koenigs embedding problem for semigroups of self-maps. The machinery developed in the study of the roots allows us to deduce in a quick way some results on embedding an LFT into such a semigroup. This has been partly known for some time but in the full generality the statement has only been completed recently and by different methods [8].

Acknowledgments. We are most grateful to Professor Christian Pommerenke for some useful comments and for calling our attention to his paper [19]. The initial proof of Theorem 4 in this paper was based on the theory of models for discrete iteration in the unit disk, the key tool being the uniqueness of solutions of the well-known Schroeder and Abel equations for certain families of holomorphic self-maps of the unit disk. However, influenced by Pommerenke's proof of [19, Theorem 3], based only on the uniqueness for the Cauchy problem for ordinary differential equations, we have been able to avoid completely the approach based on models, thus simplifying the presentation.

\section{Some BACKGROUnd}

1.1. Two simple criteria for an LFT to map the disk into itself. It is clear that only some LFT's are self-maps of the disk. However, it seems quite difficult to find a criterion explicitly stated in the literature for deciding when an LFT given by

$$
\varphi(z)=\frac{a z+b}{c z+d}, \quad a d-b c \neq 0,
$$

is a self-map of $\mathbb{D}$ in terms of $a, b, c$, and $d$. It is our belief that such criteria deserve to be mentioned explicitly since they are both simple and quite useful. We begin by stating two simple tests of this kind. The first one is from the third author's paper [17].

Proposition 1. For a map $\varphi$ given by $(1)$ we have $\varphi(\mathbb{D}) \subset \mathbb{D}$ if and only if

$$
|b \bar{d}-a \bar{c}|+|a d-b c| \leq|d|^{2}-|c|^{2} .
$$

Here is yet another criterion whose proof is simpler than the one of Proposition 1 and which is equally efficacious for our purpose. Note that, although the two criteria are clearly equivalent, it does not seem simple to deduce any of them from the other by elementary algebraic methods. 
LEMMA 1. An LFT given by (1) maps $\mathbb{D}$ into itself if and only if $|d|>|c|$ and

$$
2|a \bar{b}-c \bar{d}| \leq|c|^{2}+|d|^{2}-|a|^{2}-|b|^{2} .
$$

Moreover, $\varphi$ is a disk automorphism if and only if $|c|^{2}+|d|^{2}-|a|^{2}-|b|^{2}=$ $a \bar{b}-c \bar{d}=0$.

Proof. In order that an LFT $\varphi$ map $\mathbb{D}$ into itself, it must also map the closed disk $\overline{\mathbb{D}}$ into itself. The condition $|d|>|c|$ is clearly necessary in order for $\varphi$ to be analytic in $\overline{\mathbb{D}}$. By the Maximum Modulus Principle, the condition of being a self-map of $\overline{\mathbb{D}}$ is equivalent to $|a z+b|^{2} \leq|c z+d|^{2}$ for all $z$ of modulus one, and this happens if and only if

$$
2 \operatorname{Re}\{(a \bar{b}-c \bar{d}) z\} \leq|c|^{2}+|d|^{2}-|a|^{2}-|b|^{2}, \quad z \in \mathbb{D} .
$$

Taking the supremum over all numbers $z$ of modulus one, it is immediate that the last condition is equivalent to (3). The automorphism part is clear.

In the special case when $\varphi(0)=0$, that is, when $b=0$, both criteria become simpler. In this form, they have been stated earlier in [5] and [25, p. 203], for example.

1.2. Angular limits and derivatives. We will use $\angle$ before a limit to denote the angular (non-tangential) limit. For a given self-map $f$ of $\mathbb{D}$ and a point $p \in \partial \mathbb{D}$, if the angular limit $q=\angle \lim _{z \rightarrow p} f(z)$ also belongs to $\partial \mathbb{D}$, then the angular $\operatorname{limit} \angle \lim _{z \rightarrow p} \frac{f(z)-q}{z-p}$ exists (on the Riemann sphere $\widehat{\mathbb{C}}=\mathbb{C} \cup\{\infty\})$ and is different from zero [20, Proposition 4.13]. This limit is known as the angular derivative of $f$ at $p$. As is usual, we will denote it by $f^{\prime}(p)$.

Closely related to these notions is the concept of (angular) conformality at a point $p \in \partial \mathbb{D}$, which will play a major role in our theorems and proofs. We recall that $f \in \mathcal{H}(\mathbb{D}, \mathbb{D})$ is said to be conformal at $p \in \partial \mathbb{D}$ if the angular limits

$$
q=\angle \lim _{z \rightarrow p} f(z) \in \overline{\mathbb{D}} \text { and } \quad \angle \lim _{z \rightarrow p} \frac{f(z)-q}{z-p} \neq 0, \infty
$$

exist (see [20]). Whenever $f$ is analytic at $p$, the meaning of angular conformality coincides with the usual meaning: $f^{\prime}(p) \neq 0$.

1.3. Iteration and Denjoy-Wolff points. Denote by $\mathbb{N}$ the set of all positive integers. As is usual, we will write $f_{n}$ for the $n$-th iterate of a self-map $f$ of $\mathbb{D}$, defined inductively by $f_{1}=f$ and $f_{n+1}=f \circ f_{n}, n \in \mathbb{N}$.

It can easily be deduced from the Schwarz-Pick Lemma that a non-identity self-map $f$ of the disk can have at most one fixed point in $\mathbb{D}$. If such a unique fixed point in $\mathbb{D}$ exists, it is usually called the Denjoy-Wolff point. The iterates $f_{n}$ of $f$ converge to it uniformly on the compact subsets of $\mathbb{D}$ whenever $f$ is not a disk automorphism, but even for an automorphism 
with a unique fixed point in $\mathbb{D}$ we will still refer to such a point as the Denjoy-Wolff point of $f$.

If $f$ has no fixed point in $\mathbb{D}$, the Denjoy-Wolff theorem [14], [22] guarantees the existence of a unique point $p$ on the unit circle $\partial \mathbb{D}$ which is the attractive fixed point, that is, the iterates $f_{n}$ converge to $p$ uniformly on the compact subsets of $\mathbb{D}$. Such $p$ is again called the Denjoy-Wolff point of $f$. Note that $f$ can have other (boundary) fixed points.

Whenever $\varphi$ is a linear fractional self-map of the disk, its Denjoy-Wolff point is a true fixed point since the map is holomorphic in a disk larger than $\mathbb{D}$ and centered at the origin.

When $p \in \partial \mathbb{D}$ is the Denjoy-Wolff point of $f$, then $f^{\prime}(p)$ is actually realvalued and, moreover, $0<f^{\prime}(p) \leq 1$; see [20]. As is often done in the literature, we classify the non-identity holomorphic self-maps of the disk into three categories according to their behavior near the Denjoy-Wolff point:

(a) elliptic maps: the ones with a fixed point inside the disk;

(b) hyperbolic: the ones with the Denjoy-Wolff point $p \in \partial \mathbb{D}$ such that $f^{\prime}(p)<1$

(c) parabolic: the ones with the Denjoy-Wolff point $p \in \partial \mathbb{D}$ such that $f^{\prime}(p)=1$.

1.4. Standard simplifications by conjugation. For each of the dynamic types, our study can be normalized by conjugation with an appropriate map so as to consider instead a simplified situation, either in the disk or in the half-plane.

The standard disk automorphism $\varphi_{p}$ defined by

$$
\varphi_{p}(z)=\frac{p-z}{1-\bar{p} z}, \quad p \in \mathbb{D}
$$

interchanges the points $p$ and 0 and is its own inverse. It is easy to see that if $\varphi$ is an arbitrary elliptic automorphism of the disk different from the identity map and with Denjoy-Wolff point $p$, then

$$
\left(\varphi_{p} \circ \varphi \circ \varphi_{p}\right)(z)=\lambda z \quad \text { and } \quad \lambda=\varphi^{\prime}(p) \in \partial \mathbb{D} \backslash\{1\} .
$$

This conjugation obviously reduces the study of elliptic automorphisms to that of rotations.

The following simple and standard procedure is suitable for both the hyperbolic and parabolic maps. Let $\tau$ be the Denjoy-Wolff point of a selfmap $\varphi$ of $\mathbb{D}$ with $|\tau|=1$. The Cayley transform $T_{\tau}(z)=\frac{\tau+z}{\tau-z}$ maps $\mathbb{D}$ conformally onto the right half-plane $\mathbb{H}=\{z: \operatorname{Re} z>0\}$ and takes the point $\tau$ to infinity. Thus, to every self-map $f$ of $\mathbb{D}$ there corresponds a unique self-map $g$ of $\mathbb{H}$, called the conjugate map of $f$, such that $g=T_{\tau} \circ f \circ T_{\tau}^{-1}$ with the point at infinity as the Denjoy-Wolff point (in $\mathbb{H}$ ).

The method just described is particularly useful in the case of linear fractional maps. It is not difficult to check that every hyperbolic or parabolic linear fractional self-map $\varphi$ of $\mathbb{D}$ into itself is conjugate to a map of the form $\widetilde{\varphi}(w)=A w+B$ with $A \geq 1$ and $\operatorname{Re} B \geq 0$, with Denjoy-Wolff point at 
infinity, and with $A=1 / \varphi^{\prime}(\tau)$. Hence, $\varphi$ is parabolic if and only if $A=1$ and hyperbolic if and only if $A>1$.

\section{Conformality of SOlutions And COMPatibility of Dynamic} TYPES

In this (and the next) section we study the intertwining equation:

$$
f \circ \varphi=\psi \circ f \text {. }
$$

We first state and prove some basic necessary conditions for the existence of solutions to (5). They either tell us that the solution must be conformal at the Denjoy-Wolff point of $\varphi$ or indicate what dynamic types are required of $\varphi$ and $\psi$ in order that the solution exist.

2.1. Conformality of solutions. In what follows, $i d_{\mathbb{D}}$ will denote the identity map acting on the disk. Since a self-map of the unit disk other than $i d_{\mathbb{D}}$ has at most one fixed point in $\mathbb{D}$, if (5) holds and the fixed point $p$ of $\varphi$ belongs to $\mathbb{D}$, then $f(p)$ is a fixed point of $\psi$ in $\mathbb{D}$.

We begin by proving an auxiliary statement which may be of independent interest. It extends, and is modeled after, a similar but more special observation from [3] on the fixed points of two intertwining linear fractional maps to the boundary. It also gives some information about the (angular) conformality of $f$ which seems to be a novelty in this context.

Proposition 2. Suppose that (5) holds for the self-maps $f, \varphi$, and $\psi$ of $\mathbb{D}$, where $\varphi$ and $\psi$ are LFT's. If the Denjoy-Wolff point $p$ of $\varphi$ belongs to $\partial \mathbb{D}$ and $\psi$ is not an elliptic automorphism, then $\angle \lim _{z \rightarrow p} f(z)=q \in \overline{\mathbb{D}}$, where $q$ is the Denjoy-Wolff point of $\psi$. Moreover, if $q$ also belongs to $\partial \mathbb{D}$, then there exists $\mu \in \widehat{\mathbb{C}} \backslash\{0\}$ such that

$$
\mu=\angle \lim _{z \rightarrow p} \frac{f(z)-q}{z-p} .
$$

In particular, $f$ is conformal at $p$ if and only if $\mu \neq \infty$.

Proof. For each non-negative integer $n$, write $I_{n}=\left[1-2^{-n}, 1-2^{-(n+1)}\right)$. Define $\gamma:[0,1) \rightarrow \mathbb{D}$ by

$$
\gamma(t)=\varphi_{n}\left(\left(2^{n+1} t-\left(2^{n+1}-2\right)\right) \varphi(0)\right), \quad t \in I_{n} .
$$

Clearly, $\gamma$ is continuous in $\left(1-2^{-n}, 1-2^{-(n+1)}\right)$ for all $n$, so it is only left to check its continuity at each point of the form $1-2^{-n}$. It is obvious that

$$
\lim _{t \searrow 1-2^{-n}} \gamma(t)=\varphi_{n}(0)
$$

and also

$$
\begin{aligned}
\lim _{t \nearrow 1-2^{-n}} \gamma(t) & =\lim _{t \nearrow 1-2^{-n}} \varphi_{n-1}\left(\left(2^{n} t-\left(2^{n}-2\right)\right) \varphi(0)\right) \\
& =\varphi_{n-1}(\varphi(0))=\varphi_{n}(0)
\end{aligned}
$$

hence $\gamma$ is continuous in $[0,1)$ and so is a curve in the unit disk. 
The segment $S=[0, \varphi(0)]$ is a compact subset of $\mathbb{D}$. On the one hand, by the Denjoy-Wolff Theorem, the sequence $\left\{\varphi_{n}\right\}_{n=1}^{\infty}$ converges to $p$ uniformly on $S$ and therefore $\lim _{t \rightarrow 1} \gamma(t)=p$. On the other hand, it follows again from the Denjoy-Wolff Theorem that the sequence $\left\{\psi_{n}\right\}_{n=1}^{\infty}$ converges to $q$ uniformly on $f(S)$.

By an inductive argument, the intertwining equation (5) easily implies that $f \circ \varphi_{n}=\psi_{n} \circ f$. Thus, given $t \in I_{n}$ we have $f(\gamma(t))=f\left(\varphi_{n}(w)\right)=$ $\psi_{n}(f(w))$ for some point $w=w(t) \in[0, \varphi(0)]$. Therefore, we conclude that $\lim _{t \rightarrow 1} f(\gamma(t))=q$. Finally, by Lindelöf's classical theorem [21, $\left.§ 12.10\right]$ it follows that $\angle \lim _{z \rightarrow p} f(z)=q$.

Assume now that $q$ also belongs to $\partial \mathbb{D}$ and consider $g(z)=p \bar{q} f(z), z \in \mathbb{D}$. Clearly, $g$ is a holomorphic self-map of $\mathbb{D}$ which has $p$ as a fixed point, and the corresponding angular limit exists. Therefore, there exists $\delta \in$ $(0,+\infty) \cup\{+\infty\}$ such that

$$
\delta=\angle \lim _{z \rightarrow p} \frac{g(z)-p}{z-p} .
$$

The existence of the number $\mu$ defined in the statement follows immediately by taking $\mu=\bar{p} q \delta$.

There exists a function $f \in \mathcal{H}(\mathbb{D}, \mathbb{D})$ which is not conformal at $p$ but still satisfies the intertwining equation $f \circ \varphi=\psi \circ f$, with either both $\varphi$ and $\psi$ elliptic or both hyperbolic, as will be seen from our later Example 7 and Example 8. However, this is impossible when $\varphi$ and $\psi$ are parabolic, as the following result shows.

TheOREM 1. Suppose that (5) holds for the self-maps $f, \varphi$, and $\psi$ of $\mathbb{D}$, where $\varphi$ and $\psi$ are parabolic LFT's. Likewise, let $p$ and $q$ be the DenjoyWolff points of $\varphi$ and $\psi$, respectively. Then, $f$ is conformal at $p$ and actually

$$
p \bar{q} f^{\prime}(p) \in(0,+\infty) \quad \text { and } \quad f^{\prime}(p)=\frac{\varphi^{\prime \prime}(p)}{\psi^{\prime \prime}(q)} .
$$

Proof. By Proposition 2, in order to prove that $f$ is conformal at $p$, we need only check that $\mu \neq \infty$. We apply the procedure described in Subsection 1.4. Consider the function $g: \mathbb{H} \rightarrow \mathbb{H}$ defined as $g=T_{q} \circ f \circ T_{p}^{-1}$, where $T_{p}$ and $T_{q}$ are the associated Cayley maps with respect to $p$ and $q$. Since $g$ is a holomorphic self-map of $\mathbb{H}$, we know that there exists $c \geq 0$ such that $c=\angle \lim _{w \rightarrow \infty} \frac{g(w)}{w}$. Moreover, by the very definition of $\mu$, we have that $c p \bar{q}=1 / \mu$. So, it is only left to see that $c>0$.

By transferring the intertwining equation from $\mathbb{D}$ to $\mathbb{H}$, we find that $g \circ \widehat{\varphi}=$ $\widehat{\psi} \circ g$, where

$$
\widehat{\varphi}(w)=\left(T_{p} \circ \varphi \circ T_{p}^{-1}\right)(w)=w+a
$$

and

$$
\widehat{\psi}(w)=\left(T_{q} \circ \psi \circ T_{q}^{-1}\right)(w)=w+b,
$$


for some non-zero complex numbers $a$ and $b$ such that $\operatorname{Re} a, \operatorname{Re} b \geq 0$ and for all $w \in \mathbb{H}$. Iterating, we also obtain that, for every $w \in \mathbb{H}$ and for all $n \in \mathbb{N}$ we have

$$
g(w+n a)=g(w)+n b .
$$

From here we get that

$$
\frac{g(n+n a)}{n+n a}(1+a)=\frac{g(n)}{n}+b
$$

and since the sequences $\{n\}_{n=1}^{\infty}$ and $\{n+n a\}_{n=1}^{\infty}$ tend non-tangentially to infinity, by letting $n \rightarrow \infty$ we deduce that $c(1+a)=c+b$, hence $c a=b$. Since $b \neq 0$, we conclude that $c>0$ as desired.

A tedious but straightforward computation shows that $a=p \varphi^{\prime \prime}(p)$ and $b=q \psi^{\prime \prime}(q)$. Thus, recalling that $c a=b$, we finally have that

$$
f^{\prime}(p)=\mu=\frac{1}{c} \frac{q}{p}=\frac{p \varphi^{\prime \prime}(p)}{q \psi^{\prime \prime}(q)} \frac{q}{p}=\frac{\varphi^{\prime \prime}(p)}{\psi^{\prime \prime}(q)} .
$$

2.2. Compatibility of dynamic types of the intertwining LFT's. Our next two theorems tell us that assuming that one of the maps $\varphi$ or $\psi$ is of certain dynamic type forces the other to be of certain type (often the same) in order that the solution to (5) exist.

We will frequently use the term rational elliptic automorphism for an elliptic automorphism $\varphi$ conjugate to the map $R_{\lambda}(z)=\lambda z$, where $\lambda^{n}=1$ for some positive integer $n$. This is clearly equivalent to $\varphi$ being idempotent: $\varphi_{n}=i d_{\mathbb{D}}$ for some $n \in \mathbb{N}$. However, the term "rational elliptic automorphism" is very common in dynamics and we will use it here as well.

THEOREM 2. Let $\varphi$ and $\psi$ be linear fractional self-maps of $\mathbb{D}$ and let $f \in$ $\mathcal{H}(\mathbb{D}, \mathbb{D})$ be a non-constant function. Assume that the intertwining equation (5) holds. Then we have the following conclusions:

(a) If $\varphi=i d_{\mathbb{D}}$, then $\psi=i d_{\mathbb{D}}$.

(b) If $\psi=i d_{\mathbb{D}}$ and $\varphi \neq i d_{\mathbb{D}}$, $p$ is the Denjoy-Wolff point of $\varphi$ and there exists the angular limit $\angle \lim _{z \rightarrow p} f(z)$, then $\varphi$ is either a rational elliptic automorphism or a parabolic automorphism.

Proof. (a) This is the easy case because (5) simply reads $f=\psi \circ f$. Since $f$ is not a constant function, $f(\mathbb{D})$ is an open set and for all $w$ in this set we have $w=\psi(w)$, which shows that $\psi=i d_{\mathbb{D}}$.

(b) Let $\varphi$ be elliptic; then $p \in \mathbb{D}$. Suppose $\varphi$ is not an automorphism. On the one hand, just like earlier, (5) implies that $f \circ \varphi_{n}=f$ for all $n \in \mathbb{N}$. On the other hand, $\varphi_{n} \rightarrow p$ pointwise in $\mathbb{D}$, hence $f \equiv f(p)$ in $\mathbb{D}$. Since $f \not \equiv$ const, it follows that $\varphi$ must be an automorphism.

We will now show that $\varphi$ is rational. Write $\lambda=\varphi^{\prime}(p) \in \partial \mathbb{D}$. Set $g=$ $\varphi_{q} \circ f \circ \varphi_{p}$. Notice that $g(0)=0$ and $g(\lambda z)=g(z)$ for all $z \in \mathbb{D}$. Moreover, 
let $\sum_{n=1}^{\infty} a_{n} z^{n}$ be the Taylor series for $g$ around zero. Then

$$
a_{n} \lambda^{n}=a_{n}, \quad \text { for all } n \in \mathbb{N} .
$$

Since $f$ is not constant, the function $g$ is not identically zero and we conclude that there exists $n \in \mathbb{N}$ such that $\lambda^{n}=1$. Hence $\varphi$ is rational.

Now suppose that $\varphi$ is not elliptic and let us show that it must be a parabolic automorphism. We can transfer the equation $f \circ \varphi=f$ to the right half-plane by composing with the Cayley map associated with $p$ and its inverse as in the proof of Theorem 1 . This leads to the following equation for the corresponding self-maps $\widehat{f}, \widehat{\varphi}$ of the right half-plane: $\widehat{f} \circ \widehat{\varphi}=\widehat{f}$, where $\widehat{\varphi}(w)=a w+b$ with $a \geq 1$ and $\operatorname{Re} b \geq 0$. In the case $a>1$ it follows by iteration that

$$
\widehat{f}\left(a^{n} w+\frac{a^{n}-1}{a-1} b\right)=\widehat{f}(w), \quad w \in \mathbb{H} .
$$

Since $\lim _{n \rightarrow \infty} a^{n} w+\frac{a^{n}-1}{a-1} b=\infty$ non-tangentially and the angular limit $\angle \lim _{z \rightarrow p} f(z)$ exists (and hence so does $\angle \lim _{w \rightarrow \infty} \widehat{f}(w)$ ), it follows that $f \equiv$ const.

Thus, in order that a non-trivial solution to (5) exist, we must have $a=1$, that is, $\varphi$ must be parabolic. If $\operatorname{Re} b>0$ then $w+n b \rightarrow \infty$ non-tangentially. Again, by (5) we have that $\widehat{f}(w+n b)=\widehat{f}(w)$ for all $w \in \mathbb{H}$ and all $n \in \mathbb{N}$ and once more it follows that $f$ is constant. Therefore $\operatorname{Re} b=0$, so $\varphi$ is a parabolic automorphism.

Both situations referred to in part (b) of Theorem 2 are actually possible.

EXAmple 1. Let $\varphi(z)=-z$ and $f(z)=z^{2}$. Then $\varphi$ is a rational elliptic automorphism with $n=2$ and $f=f \circ \varphi$.

EXAMPLE 2. Let

$$
\varphi(z)=\frac{2 \pi i+(1-2 \pi i) z}{1+2 \pi i-2 \pi i z}, \quad f(z)=e^{-\frac{1}{2} \frac{1+z}{1-z}} .
$$

Then $\varphi$ is a parabolic automorphism with Denjoy-Wolff point $p=1$ and $f=f \circ \varphi$ holds in $\mathbb{D}$.

Note also that without the assumption on the existence of the angular limit in (b) the result no longer holds, as the following example shows.

EXAMPLE 3. Consider the hyperbolic non-automorphic map $\varphi(z)=(1+$ $z) / 2$. Taking the principal branch of logarithm restricted to the right halfplane, define

$$
f(z)=\frac{1}{2} \exp \left(-\frac{\pi^{2}}{\log 2}\right) \exp \left(\frac{2 \pi i}{\log 2} \log \frac{1}{1-z}\right) .
$$

The function $f$ is easily seen to map the disk onto a compact subset of $\mathbb{D}$ and is therefore an elliptic self-map of $\mathbb{D}$. It is also readily verified that $f \circ \varphi=f$. 
Before stating further results, let us recall that the hyperbolic metric in the disk is defined by

$$
\varrho(z, w)=\frac{1}{2} \log \frac{1+\left|\varphi_{w}(z)\right|}{1-\left|\varphi_{w}(z)\right|}=\inf _{\gamma} \int_{\gamma} \frac{|d \zeta|}{1-|\zeta|^{2}},
$$

taking the infimum over all rectifiable curves $\gamma$ in $\mathbb{D}$ from $z$ to $w$.

Given a holomorphic self-map $g$ of $\mathbb{D}$ and a point $z_{0}$ in $\mathbb{D}$, we define the forward orbit of $z_{0}$ under $g$ as the sequence $z_{n}=g_{n}\left(z_{0}\right)$. It is customary to say that $g$ is of zero hyperbolic step if for some point $z_{0}$ its iterations $z_{n}=g_{n}\left(z_{0}\right)$ satisfy the condition $\lim _{n \rightarrow \infty} \varrho\left(z_{n}, z_{n+1}\right)=0$. It is well-known that the word "some" here can be replaced by "all". In other words, the definition does not depend on the choice of the initial point of the orbit.

Using the Schwarz-Pick Lemma, it is easy to see that the maps which are not of zero hyperbolic step are precisely those holomorphic self-maps $\varphi$ of $\mathbb{D}$ for which

$$
\lim _{n \rightarrow \infty} \varrho\left(z_{n}, z_{n+1}\right)>0,
$$

for some forward orbit $\left\{z_{n}\right\}_{n=1}^{\infty}$ of $g$, and hence for all such orbits. This is the reason why they are called maps of positive hyperbolic step. For a survey of these properties, the reader may consult [11].

It is easy to show that if $g$ is elliptic and is not an automorphism, then it is of zero hyperbolic step. If $g$ is hyperbolic, then it is of positive hyperbolic step. The following dichotomy holds for parabolic linear fractional maps: every parabolic automorphism of $\mathbb{D}$ is of positive hyperbolic step, while all non-automorphic, linear fractional, parabolic self-maps of $\mathbb{D}$ are of zero hyperbolic step.

THEOREM 3. Let $\varphi, \psi$ be two linear fractional self-maps of $\mathbb{D}$, both different from the identity, and let $f \in \mathcal{H}(\mathbb{D}, \mathbb{D})$ be conformal at $p$, where $p$ is the Denjoy-Wolff point of $\varphi$. Assume that the intertwining equation $f \circ \varphi=\psi \circ f$ holds. Then:

(a) If $\varphi$ is elliptic non-automorphic, then $\psi$ is also elliptic non-automorphic.

(b) If $\psi$ is elliptic non-automorphic, then the function $\varphi$ is either a hyperbolic map or an elliptic non-automorphic map.

(c) The map $\varphi$ is an elliptic automorphism if and only if $\psi$ is also an elliptic automorphism.

(d) If $\psi$ is hyperbolic, then $\varphi$ is also hyperbolic.

(e) If $\varphi$ is hyperbolic, then $\psi$ is either a hyperbolic or an elliptic nonautomorphic map. Moreover, if $\varphi$ is a hyperbolic automorphism, then $\psi$ is either a hyperbolic automorphism or an elliptic non-automorphic map such that $\overline{\psi(\mathbb{D})} \cap \partial \mathbb{D} \neq \emptyset$.

(f) $\varphi$ is parabolic non-automorphic if and only if $\psi$ is also parabolic non-automorphic.

(g) $\varphi$ is a parabolic automorphism if and only if $\psi$ is also a parabolic automorphism. 
Proof. In what follows, $q$ will always denote the Denjoy-Wolff point of $\psi$.

(a) Like in the discussion at the beginning of Subsection 2.1, we see that $\psi$ must also be elliptic and $q=f(p) \in \mathbb{D}$. Moreover, $f^{\prime}(p) \varphi^{\prime}(p)=\psi^{\prime}(q) f^{\prime}(p)$. Since $f^{\prime}(p) \neq 0$, we conclude that $\left|\psi^{\prime}(q)\right|=\left|\varphi^{\prime}(p)\right|<1$. Therefore, $\psi$ is an elliptic and non-automorphic map.

(b) We will show that $\varphi$ is neither a parabolic map nor an elliptic automorphism.

If $\varphi$ were parabolic, applying Proposition 2, the chain rule for the angular derivative and conformality of $f$ at $p$, we would obtain $\psi^{\prime}(q)=\varphi^{\prime}(p)=1$, which is impossible because $\psi$ is elliptic and non-automorphic. Assuming that $\varphi$ is an elliptic automorphism, one can apply a completely analogous but easier argument to get a contradiction.

(c) We first prove the forward implication. This case is more delicate than the previous ones because our proof will not use the conformality of $f$ at $p$. (Note that with that assumption a much simpler proof is possible.) Since $\varphi$ is elliptic, $\psi$ is also such. Let us prove that $\psi$ is also an automorphism. We have already established earlier that $f(p)=q$. Consider the function $g=f \circ \varphi_{p}$. Then $g(\lambda z)=\psi(g(z))$ for all $z$. It readily follows by induction that $g\left(\lambda^{n} z\right)=\psi_{n}(g(z))$ for all positive integers $n$ and all $z \in \mathbb{D}$. Observe also that $g(0)=q$.

Suppose that $\psi$ is not an automorphism. Then $\psi_{n} \rightarrow q$ pointwise. Thus, for each $z \in \mathbb{D}$,

$$
\left|g\left(\lambda^{n} z\right)-g(0)\right|=\left|\psi_{n}(g(z))-q\right| \rightarrow 0 \quad \text { as } \quad n \rightarrow \infty .
$$

Since $|\lambda|=1$, by continuity and a basic compactness argument it is easy to see that for each $r \in(0,1)$, there is a point $\xi \in \mathbb{D}$, with $|\xi|=r$, such that $g(\xi)=g(0)$. Therefore, $g$ is constant, hence so is $f$, which contradicts our assumption. Thus, $\psi$ is an automorphism.

Now for the reverse implication. Suppose that $\varphi$ is not elliptic. Choose a sequence $\left\{z_{n}\right\}_{n=1}^{\infty}$ in the unit disk that converges to $p$ non-tangentially. Then also $\varphi\left(z_{n}\right) \rightarrow p$ non-tangentially and, using the conformality of $f$ at $p$, we conclude that the sequence $\left\{f\left(\varphi\left(z_{n}\right)\right)\right\}_{n=1}^{\infty}$ is convergent to some point $\widehat{q}$. Moreover, the sequence $\left\{f\left(z_{n}\right)\right\}_{n=1}^{\infty}$ also tends to $\widehat{q}$, hence $\psi(\widehat{q})=\widehat{q}$. Since $\widehat{q} \in \overline{\mathbb{D}}$ and $\psi$ is an elliptic automorphism, we conclude that $\widehat{q}$ is the DenjoyWolff point of $\psi$. Now, applying the chain rule in the intertwining equation (5) and using again the conformality of $f$ at $p$, we get that $\varphi^{\prime}(p)=\psi^{\prime}(q) \in$ $\partial \mathbb{D} \backslash\{1\}$, which contradicts our assumption that $\varphi$ is either hyperbolic or parabolic. Therefore, $\varphi$ is elliptic.

Once we know that $\varphi$ is elliptic, we just apply the chain rule in the intertwining equation together with the conformality of $f$ at $p$ to obtain that $\varphi^{\prime}(p)=\psi^{\prime}(q) \in \partial \mathbb{D} \backslash\{1\}$. Thus, $\varphi$ is an automorphism.

(d) We know that

$$
\varrho\left(\varphi_{n}(0), \varphi_{n+1}(0)\right) \geq \varrho\left(f\left(\varphi_{n}(0)\right), f\left(\varphi_{n+1}(0)\right)\right)=\varrho\left(\psi_{n}(f(0)), \psi_{n+1}(f(0))\right) .
$$


Since $\psi$ is a hyperbolic linear fractional map, the sequence

$$
\left\{\varrho\left(\psi_{n}(f(0)), \psi_{n+1}(f(0))\right)\right\}_{n=1}^{\infty}
$$

converges to a positive real number. Therefore, $\varphi$ is of positive hyperbolic step and this implies that $\varphi$ is either an elliptic automorphism, a parabolic automorphism, or a hyperbolic map. It cannot be elliptic since $f(p)=q$ and $\psi$ is not elliptic. Bearing in mind Proposition 2 and using the chain rule for the angular derivative, we obtain that $f^{\prime}(p) \varphi^{\prime}(p)=\psi^{\prime}(q) f^{\prime}(p)$. Since $f$ is conformal at $p$, we find that $\varphi^{\prime}(p)=\psi^{\prime}(q) \in(0,1)$. In particular, $\varphi$ is not parabolic and, therefore, it is hyperbolic.

(e) This case seems to require the most involved proof by far. We first observe that $\psi$ can neither be a parabolic map nor an elliptic automorphism.

If $\psi$ were a parabolic map, then applying Proposition 2, the chain rule for the angular derivative, and conformality of $f$ at $p$, we would obtain that $\varphi^{\prime}(p)=\psi^{\prime}(q)=1$, which is absurd because $\varphi$ is hyperbolic.

Suppose now $\psi$ is an elliptic automorphism. By assumption, $f$ is conformal at $p$, we know that there exists $\widehat{q}=\angle \lim _{z \rightarrow p} f(z) \in \overline{\mathbb{D}}$. Since $\varphi$ maps non-tangential regions at $p$ into non-tangential regions at $p$, we deduce that $\psi(\widehat{q})=\widehat{q}$. Given that $\psi$ has only one fixed point in $\overline{\mathbb{D}}$, it follows that $\widehat{q}=q$. Therefore, by the Denjoy-Wolff theorem

$$
\lim _{n \rightarrow \infty}\left|f\left(\varphi_{n}(0)\right)\right|=|q|=1=\lim _{n \rightarrow \infty}\left|\psi_{n}(f(0))\right| .
$$

However, since $\psi$ is an elliptic automorphism of $\mathbb{D}$, hence it is a rotation in hyperbolic metric and so $\sup _{n \in \mathbb{N}}\left|\psi_{n}(f(0))\right|<1$, which is impossible.

We have thus shown that $\psi$ must be either a hyperbolic map or an elliptic non-automorphic map.

Our next step will be to show that if $\varphi$ is a hyperbolic automorphism and $\psi$ is an elliptic non-automorphic map, then $\overline{\psi(\mathbb{D})} \cap \partial \mathbb{D} \neq \emptyset$. Suppose, on the contrary, that $\overline{\psi(\mathbb{D})} \subset \mathbb{D}$. Denote by $q$ the Denjoy-Wolf point of $\psi$. Then replacing $\psi$ by the function $\varphi_{q} \circ \psi \circ \varphi_{q}$ and $f$ by $\varphi_{q} \circ f$ if necessary, we may assume that $q=0$. In this case, we have $\psi(z)=\frac{a z}{c z+1}$ and, by a special case of Proposition 1 or Lemma 1, we must have $|a|+|c| \leq 1$. Obviously, $a=\psi^{\prime}(0)$ and then $0<|a|<1$. By conformality of $f$ at $p$, deriving the intertwining equation at the point $p$, we obtain $0<a<1$. If $a+|c|=1$, then

$$
\psi\left(-\frac{\bar{c}}{|c|}\right)=-\frac{\bar{c}}{|c|} \in \partial \mathbb{D}
$$

This contradicts the assumption that $\overline{\psi(\mathbb{D})} \subset \mathbb{D}$, hence $a+|c|<1$. Write

$$
\sigma(z)=\frac{z}{C z+1}, \quad \text { where } C=c /(1-a) .
$$

From $a+|c|<1$ it follows that $|C|<1$, so $\sigma$ is bounded on the unit disk. Moreover, $\sigma \circ \psi=a \sigma$. Define $g:=\sigma \circ f$. Then $g$ is an analytic and bounded function in the unit disk and $g \circ \varphi=a g$. Since $\varphi$ is an automorphism, we 
have

$$
\sup \{|g(z)|: z \in \mathbb{D}\}=\sup \{|g \circ \varphi(z)|: z \in \mathbb{D}\}=a \sup \{|g(z)|: z \in \mathbb{D}\} .
$$

But $a<1$ and $g$ is bounded, hence $g(z)=0$ for all $z \in \mathbb{D}$. This implies that $f(z)=0$, for all $z \in \mathbb{D}$. In other words, $f$ is constant, which is in contradiction with our assumptions.

It only remains to show that if $\varphi$ is an hyperbolic automorphism and $\psi$ is also hyperbolic, then $\psi$ is also an automorphism. Again, let us denote by $q \in \partial \mathbb{D}$ the corresponding Denjoy-Wolf point of $\psi$ (now, $\psi^{\prime}(q) \in(0,1)$ ). Consider the rotation $r(z)=p \bar{q} z, z \in \mathbb{D}$ that maps $q$ to $p$ (note that $r^{-1}(z)=\bar{p} q z$ is also a rotation) and define $h:=r \circ f$ and $\widehat{\psi}:=r \circ \psi \circ r^{-1}$. A straightforward computation shows that $\widehat{\psi}$ is a hyperbolic map with DenjoyWolff point $p$ such that $\widehat{\psi}^{\prime}(p)=\psi^{\prime}(q)$ and

$$
h \circ \varphi=\widehat{\psi} \circ h .
$$

Moreover, by the conformality of $f$ at $p$, we see that $\widehat{\psi}^{\prime}(p)=\varphi^{\prime}(p)$.

From this point on, we change the setting from $\mathbb{D}$ to $\mathbb{H}$ and consider the analytic functions in the right half-plane given by $H:=T_{p} \circ h \circ T_{p}^{-1}, \Phi:=$ $T_{p} \circ \varphi \circ T_{p}^{-1}, \Psi:=T_{p} \circ \widehat{\psi} \circ T_{p}^{-1}$. Bearing in mind that $\widehat{\psi}^{\prime}(p)=\varphi^{\prime}(p)$, we see that there exist $\alpha>1, b, A_{1}$, and $A_{2} \in \mathbb{R}$ such that $b \neq 0, A:=A_{1}+i A_{2} \neq 0$ and $A_{1} \geq 0$ such that

$$
H(\alpha w+i b)=\alpha H(w)+A, \quad \text { for all } w \in \mathbb{H} .
$$

In order to show that $\Psi$ is an automorphism of the right half-plane and thus complete the proof, we need only show that $A_{1}=0$.

Iterating, we obtain

$$
H\left(\alpha^{n} w+i b \frac{\alpha^{n}-1}{\alpha-1}\right)=\alpha^{n} H(w)+A \frac{\alpha^{n}-1}{\alpha-1}
$$

for all $n \in \mathbb{N}$ and for all $w \in \mathbb{H}$. Note that, for all $w \in \mathbb{H}$, the sequence $\left\{\alpha^{n} w+i b \frac{\alpha^{n}-1}{\alpha-1}\right\}_{n=1}^{\infty}$ tends non-tangentially to $\infty$. Since $H$ is an analytic self-map of $\mathbb{H}$, by Wolff's well-known theorem (see, for example, [14, p. 60, Exercise 2.3.10 (b)]), there exists a constant $c \geq 0$ such that

$$
c=\angle \lim _{w \rightarrow \infty} \frac{H(w)}{w}=\lim _{n \rightarrow \infty} \frac{H\left(\alpha^{n} w+i b \frac{\alpha^{n}-1}{\alpha-1}\right)}{\alpha^{n} w+i b \frac{\alpha^{n}-1}{\alpha-1}} .
$$

Therefore, for all $w \in \mathbb{H}$,

$$
c=\lim _{n \rightarrow \infty} \frac{\alpha^{n} H(w)+A \frac{\alpha^{n}-1}{\alpha-1}}{\alpha^{n} w+i b \frac{\alpha^{n}-1}{\alpha-1}}=\lim _{n \rightarrow \infty} \frac{H(w)+A \frac{1-\alpha^{-n}}{\alpha-1}}{w+i b \frac{1-\alpha^{-n}}{\alpha-1}}=\frac{H(w)+\frac{A}{\alpha-1}}{w+\frac{i b}{\alpha-1}} .
$$

Hence,

$$
H(w)=c w+\frac{c b}{\alpha-1} i-\frac{A}{\alpha-1}, w \in \mathbb{H} .
$$


Now, since $H$ is a self-map of $\mathbb{H}$, we conclude that

$$
0 \leq \operatorname{Re}\left(\frac{c b}{\alpha-1} i-\frac{A}{\alpha-1}\right)=-\frac{A_{1}}{\alpha-1} .
$$

Our assumption $\alpha>1$ tells us that $A_{1} \leq 0$. We already know that $A_{1} \geq 0$, hence $A_{1}=0$, and we are done.

(f) We first prove the forward implication. Recall that a parabolic linear fractional map which is not an automorphism is of zero hyperbolic step. Thus, given $z \in \mathbb{D}$, we have that $\varrho\left(\varphi_{n}(0), \varphi_{n+1}(0)\right) \rightarrow 0$ as $n \rightarrow \infty$. Hence

$$
\varrho\left(\varphi_{n}(0), \varphi_{n+1}(0)\right) \geq \varrho\left(f\left(\varphi_{n}(0)\right), f\left(\varphi_{n+1}(0)\right)\right)=\varrho\left(\psi_{n}(f(0)), \psi_{n+1}(f(0))\right) .
$$

Therefore, $\lim _{n \rightarrow \infty} \varrho\left(\psi_{n}(f(0)), \psi_{n+1}(f(0))\right)=0$, which shows that $\psi$ is of zero hyperbolic step. Thus, $\psi$ is also a parabolic or an elliptic linear fractional map different from an automorphism. Bearing in mind statement (b), we conclude that $\psi$ is parabolic non-automorphic.

Now for the reverse implication. From the statements (a), (c), and (e), we know that $\varphi$ is parabolic, so we only have to prove that it is nonautomorphic. Applying Theorem 1 we deduce that

$$
q \psi^{\prime \prime}(q) f^{\prime}(p) \frac{p}{q}=p \varphi^{\prime \prime}(p)
$$

and $\operatorname{Re} q \psi^{\prime \prime}(q)>0$ if and only if $\operatorname{Re} p \varphi^{\prime \prime}(p)>0$. By recalling the following general fact about an arbitrary linear fractional parabolic self-map $\varphi$ of $\mathbb{D}$ : if $r$ denotes its Denjoy-Wolff point, then always $\operatorname{Re} r \varphi^{\prime \prime}(r) \geq 0$ and $\varphi$ is an automorphism if and only if $\operatorname{Re} r \varphi^{\prime \prime}(r)=0$, the proof is complete.

Part (g) is a trivial consequence of the remaining six statements.

Observe that in parts (c) and (g) the maps $\varphi$ and $\psi$ must have the same type. One would expect a similar statement to hold for hyperbolic automorphisms. However, this is false.

EXAMPLE 4. Let

$$
f(z)=\frac{i z+1}{-z+2+i}, \quad \varphi(z)=\frac{z+1}{2}, \quad \psi(z)=\frac{(2+i) z+i}{z+1+2 i} .
$$

Then the intertwining equation holds, $\varphi$ is hyperbolic and non-automorphic, while $\psi$ is a hyperbolic automorphism.

Theorem 3 shows a great degree of symmetry concerning the dynamical types of $\varphi$ and $\psi$ in the non-automorphic cases as well, except for the statements (b) and (e) where a mixture of types is allowed. The following examples tell us that indeed all combinations are possible.

EXAMPLE 5. Consider the mappings

$$
f(z)=(1-z) / 2, \quad \varphi(z)=(1+z) / 2, \quad \psi(z)=z / 2, \quad z \in \mathbb{D} .
$$

Then $\varphi$ is hyperbolic and non-automorphic with Denjoy-Wolff point 1 and $\psi$ is elliptic non-automorphic with Denjoy-Wolff point 0. 
It should also be mentioned that even if $\varphi$ is a hyperbolic automorphism, $\psi$ can be an elliptic map.

EXAMPLE 6. Let

$$
\varphi(z)=\frac{3 z+1}{z+3}, \quad \psi(z)=\frac{z}{2-z}, \quad f(z)=\frac{1-z}{z+3} \quad \text { for all } z \in \mathbb{D} .
$$

Then $\varphi$ is a hyperbolic automorphism, $\psi$ is elliptic, and $f \circ \varphi=\psi \circ f$. Of course, in accord with Theorem $3, \overline{\psi(\mathbb{D})} \cap \partial \mathbb{D}=\{1\} \neq \emptyset$.

Conformality is not used to the full extent in all cases in Theorem 3. For instance, in the forward implication in (c), it is not needed at all.

\section{A RIgidity PRINCIPLE AND FURTher RESUlts ON THE INTERTWINING EQUATION}

The reader should be warned that (5) does not always imply that $f$ must be an LFT (that is, we do not necessarily have the rigidity principle here), as the following example shows.

EXAMPLE 7. Let $\varphi(z)=z / 2, \psi(z)=z / 4$, and $f(z)=z^{2}$. It is clear that $f \circ \varphi=\psi \circ f$ but $f$ is not and LFT; it is not even univalent.

Thus, again some additional conditions on $f$ (e.g., local univalence near the Denjoy-Wolff point of $\varphi$ ) should be required in order to get the rigidity. We now analyze all possible cases.

3.1. A rigidity principle for intertwining. The following statement covers the majority of the possible cases regarding the dynamical type of $\varphi$. The remaining two automorphic cases shall be dealt with in separate subsections.

ThEOREM 4. Let $\varphi$ be a linear fractional self-map of $\mathbb{D}$ which is either elliptic and non-automorphic, parabolic and non-automorphic, or hyperbolic. Let $p$ be the Denjoy-Wolff point of $\varphi$ and $f$ a self-map of $\mathbb{D}$ conformal at $p$. If (5) holds for some linear fractional self-map $\psi$ of $\mathbb{D}$ then $\psi \neq i d_{\mathbb{D}}$ and $f$ is also a linear fractional map.

Proof. First of all, observe that if (5) is satisfied then part (b) of Theorem 2 tells us that the case when $\psi$ is the identity map is excluded here.

For the sake of simplicity, suppose that the map $\varphi$ is either parabolic and non-automorphic or hyperbolic. When $\varphi$ is elliptic and non-automorphic, the proof carries through by replacing everywhere the words "converges nontangentially" by "converges".

Since the sequence $\left(\varphi_{n}(0)\right)$ converges non-tangentially to the point $p$, it follows that $f^{\prime}\left(\varphi_{n}(0)\right) \rightarrow f^{\prime}(p)$ as $n \rightarrow \infty$.

Let

where

$$
\varphi_{n}(z)=\frac{\alpha_{n} z+\beta_{n}}{\gamma_{n} z+\delta_{n}}, \quad \psi_{n}(z)=\frac{a_{n} z+b_{n}}{c_{n} z+d_{n}}
$$

$$
\alpha_{n} \delta_{n}-\beta_{n} \gamma_{n}=1, \quad a_{n} d_{n}-b_{n} c_{n}=1
$$


Differentiation of $f \circ \varphi_{n}=\psi_{n} \circ f$ yields $f^{\prime}\left(\varphi_{n}(z)\right) \varphi_{n}^{\prime}(z)=\psi_{n}^{\prime}(f(z)) f^{\prime}(z)$ and therefore

$$
\frac{f^{\prime}\left(\varphi_{n}(z)\right)}{\left(\gamma_{n} z+\delta_{n}\right)^{2}}=\frac{f^{\prime}(z)}{\left(c_{n} f(z)+d_{n}\right)^{2}}
$$

for all $n$ and for all $z \in \mathbb{D}$.

Write $c_{n}^{*}:=\frac{c_{n}}{\left|c_{n}\right|+\left|d_{n}\right|}, d_{n}^{*}:=\frac{d_{n}}{\left|c_{n}\right|+\left|d_{n}\right|}, \gamma_{n}^{*}:=\frac{\gamma_{n}}{\left|\gamma_{n}\right|+\left|\delta_{n}\right|}$, and $\delta_{n}^{*}:=\frac{\delta_{n}}{\left|\gamma_{n}\right|+\left|\delta_{n}\right|}$. By passing to convergent subsequences if necessary, we obtain $c_{n_{k}}^{*} \rightarrow c^{*}$, $d_{n_{k}}^{*} \rightarrow d^{*}, \delta_{n_{k}}^{*} \rightarrow \delta^{*}$, and $\gamma_{n_{k}}^{*} \rightarrow \gamma^{*}$, where $c^{*}, d^{*}, \gamma^{*}, \delta^{*} \in \overline{\mathbb{D}}$. Since $\left|c_{n}^{*}\right|+$ $\left|d_{n}^{*}\right|=1$ and $\left|c_{n}^{*}\right|<\left|d_{n}^{*}\right|$ for all $n$, we also get $\left|c^{*}\right|+\left|d^{*}\right|=1$ and $\left|c^{*}\right| \leq\left|d^{*}\right|$. Similarly, $\left|\gamma^{*}\right|+\left|\delta^{*}\right|=1$ and $\left|\gamma^{*}\right| \leq\left|\delta^{*}\right|$. Therefore, $\gamma^{*} z+\delta^{*} \neq 0$ and $c^{*} z+d^{*} \neq 0$ for all $z \in \mathbb{D}$.

Choose $z_{0} \in \mathbb{D}$ such that $f\left(z_{0}\right) \neq 0$ and $f^{\prime}\left(z_{0}\right) \neq 0$. By $(6), f^{\prime}\left(\varphi_{n}\left(z_{0}\right)\right) \neq 0$ for all $n$. Using (6) for $z$ and $z_{0}$, we have

$$
\frac{f^{\prime}\left(\varphi_{n}(z)\right)}{f^{\prime}\left(\varphi_{n}\left(z_{0}\right)\right)}\left(\frac{\gamma_{n} z_{0}+\delta_{n}}{\gamma_{n} z+\delta_{n}}\right)^{2}=\frac{f^{\prime}(z)}{f^{\prime}\left(z_{0}\right)}\left(\frac{c_{n} f\left(z_{0}\right)+d_{n}}{c_{n} f(z)+d_{n}}\right)^{2} \text {. }
$$

Thus

$$
\frac{f^{\prime}\left(\varphi_{n}(z)\right)}{f^{\prime}\left(\varphi_{n}\left(z_{0}\right)\right)}\left(\frac{\gamma_{n}^{*} z_{0}+\delta_{n}^{*}}{\gamma_{n}^{*} z+\delta_{n}^{*}}\right)^{2}=\frac{f^{\prime}(z)}{f^{\prime}\left(z_{0}\right)}\left(\frac{c_{n}^{*} f\left(z_{0}\right)+d_{n}^{*}}{c_{n}^{*} f(z)+d_{n}^{*}}\right)^{2}
$$

Replacing $n$ by $n_{k}$, recalling that $f$ is conformal at $p$ and taking limits, we get

$$
\left(\frac{\gamma^{*} z_{0}+\delta^{*}}{\gamma^{*} z+\delta^{*}}\right)^{2}=\frac{f^{\prime}(z)}{f^{\prime}\left(z_{0}\right)}\left(\frac{c^{*} f\left(z_{0}\right)+d^{*}}{c^{*} f(z)+d^{*}}\right)^{2} .
$$

That is, there are complex numbers $A, B, C$, and $D$ with $|C| \leq|D|$ such that

$$
f^{\prime}(z)=\left(\frac{A f(z)+B}{C z+D}\right)^{2} \quad \text { for all } \quad z \in \mathbb{D} .
$$

Let $a=f(0)$ and consider $g=\varphi_{a} \circ f$. A simple computation shows that there exist $A^{*}, B^{*}$ such that

$$
g^{\prime}(z)=\left(\frac{A^{*} g(z)+B^{*}}{C z+D}\right)^{2} \quad \text { for all } \quad z \in \mathbb{D} .
$$

That is, $g$ is the unique solution of the Cauchy problem $y^{\prime}=\left(\frac{A^{*} y+B^{*}}{C z+D}\right)^{2}$ with $y(0)=0$. Thus, $g(z)=\frac{B^{* 2} z}{\left(D C-B^{*} A^{*}\right) z+D}$. Since $f=\varphi_{a} \circ g$, it follows that $f$ must also be a linear fractional map.

A few comments and examples are in order. Our earlier Example 7 tells us that, in the elliptic case, the assumption $f^{\prime}(p) \neq 0$ is essential in order for the conclusion of the theorem to hold. Moreover, the assumption $f^{\prime}(p) \neq \infty$ cannot be omitted when the Denjoy-Wolff point of $\varphi$ is on the boundary of the unit disk. 
EXAMPLE 8. Let

$$
\varphi(z)=\frac{5 z+3}{3 z+5}, \quad \psi(z)=\frac{3 z+1}{z+3}, \quad f(z)=\frac{\sqrt{\frac{1+z}{1-z}}-1}{\sqrt{\frac{1+z}{1-z}}+1} .
$$

It can be checked that (5) holds, the Denjoy-Wolff point of $\varphi$ is $p=1$, while $\angle \lim _{z \rightarrow 1} f(z)=1$ and $f^{\prime}(1)=\infty$, even though $f$ is not an LFT.

The above example is easier to understand after transferring all the maps to the right half-plane by means of conjugation $g=T \circ f \circ T^{-1}$ via the Cayley map $T(z)=(1+z) /(1-z)$. In the right half-plane (5) becomes simply $g(4 w)=2 g(w)$, where $g(w)=\sqrt{w}$ is the map that corresponds to $f$ with an appropriately defined branch of the square root. Notice that in this example the function $\varphi$ is hyperbolic and, by Theorem 1, we know that this situation cannot occur when $\varphi$ and $\psi$ are parabolic.

The following example shows why it was important to exclude the elliptic automorphisms from Theorem 4.

EXAMPLE 9. Consider the following self-maps of the disk:

$$
\varphi(z)=\psi(z)=-z, \quad f(z)=\frac{z+z^{3}}{2} .
$$

Note that $f(\mathbb{D}) \subset \mathbb{D}, p=0, f^{\prime}(0) \neq 0$, and (5) still holds, even though $f$ is clearly not an LFT.

We also point out that there is a self-map of $\mathbb{D}$ which commutes with a parabolic automorphism but is far from being an LFT. This has been known for some time [1, Proposition 1.2.6].

3.2. The case when $\varphi$ is an elliptic automorphism. The following characterization seems to be new, in the sense that it generalizes Proposition 1.2.26 from [1]. It should be remarked that similar questions for intertwining were raised explicitly and studied in [18] in the context of the classical semi-conjugation. The reader should note the dichotomy between the conjugations related to the roots of unity and all the remaining ones.

THEOREM 5. Let $\varphi$ be an elliptic automorphism (different from the identity) with Denjoy-Wolff point $p$ and $\lambda=\varphi^{\prime}(p)$. Let $\psi$ be an arbitrary linear fractional self-map of $\mathbb{D}$. Suppose $f$ is a non-constant self-map of $\mathbb{D}$ such that $f \circ \varphi=\psi \circ f$ and define $n_{0}=\min \left\{n \in \mathbb{N}: f^{(n)}(p) \neq 0\right\}$.

(i) If $\psi$ is the identity map and $m_{0}=\min \left\{n \geq 1: \lambda^{n}=1\right\}$ as in the proof of Theorem 2, then there is a non-constant self-map of the unit disk $g$, with $g(0)=0$, such that

$$
\left(\varphi_{f(p)} \circ f \circ \varphi_{p}\right)(z)=g\left(z^{m_{0}}\right), \quad z \in \mathbb{D} .
$$

Moreover, the function $f$ is never a linear fractional map.

(ii) Assume $\psi$ is not the identity map, $q$ is its Denjoy-Wolff point, and $\mu=\psi^{\prime}(q)$. Then $\lambda^{n_{0}}=\mu$. Also, the following dichotomy takes place: 
(a) If $\lambda^{n} \neq 1$ for every positive integer $n$, then there exists a point $\beta$ in $\overline{\mathbb{D}} \backslash\{0\}$ such that $\left(\varphi_{q} \circ f \circ \varphi_{p}\right)(z)=\beta z^{n_{0}}$ for all $z \in \mathbb{D}$. In particular, $f$ is a linear fractional map if and only if $n_{0}=1$.

(b) If $\lambda^{n}=1$ for some positive integer $n$, and the integer $m_{0}$ is defined as above, then there exists a non-zero self-map $g$ of $\mathbb{D}$ such that

$$
\left(\varphi_{q} \circ f \circ \varphi_{p}\right)(z)=z^{n_{0}} g\left(z^{m_{0}}\right), \quad z \in \mathbb{D} .
$$

In particular, $f$ is a linear fractional map if and only if $g$ is constant and $n_{0}=1$.

Proof. Keeping in mind Theorem 2, one sees that the proof of statement (i) is similar to the proof of part (b) in statement (ii) by setting $n_{0}=0$. Therefore, we will only present in detail the proof of the latter case. It is easy to fill in the corresponding arguments in (i).

Since $\varphi$ is an elliptic automorphism, it follows by part (c) of Theorem 3 that $\psi$ is also such. (As remarked in the proof of that part of the theorem, the assumption on conformality of $f$ is not needed precisely in this implication.)

Each of the maps $\varphi_{p} \circ \varphi \circ \varphi_{p}$ and $\varphi_{q} \circ \psi \circ \varphi_{q}$ is a disk automorphism and fixes the origin, hence a rotation: $\varphi_{p}\left(\varphi\left(\varphi_{p}(z)\right)\right)=\lambda z$ and $\varphi_{q}\left(\psi\left(\varphi_{q}(z)\right)\right)=\mu z$, for the values $\lambda, \mu$ as defined in the statement of the theorem and for all $z$ in $\mathbb{D}$. Considering the self-map $h=\varphi_{q} \circ f \circ \varphi_{p}$ of $\mathbb{D}$ which fixes the origin, the intertwining equation implies that $h(\lambda z)=\mu h(z)$ for all $z$ in the disk. Let

$$
h(z)=\sum_{n=n_{0}}^{\infty} a_{n} z^{n}
$$

with $a_{n_{0}} \neq 0$. Thus we have $a_{n} \lambda^{n}=a_{n} \mu$ for all $n \geq n_{0}$. In particular, $\lambda^{n_{0}}=\mu$ (because $a_{n_{0}} \neq 0$ ), which completes the proof of the first part of the assertion.

We now consider the two cases corresponding to the statements (a) and (b). Observe first that if $a_{n} \neq 0$ then $\lambda^{n}=\mu=\lambda^{n_{0}}$. If there is no $m \neq 0$ such that $\lambda^{m}=1$, then $n=n_{0}$ since $\lambda^{n-n_{0}}=1$. This implies that $h(z)=a_{n_{0}} z^{n_{0}}$, with $0<\left|a_{n_{0}}\right| \leq 1$ because $h$ is a self-map of the unit disk. This implies (a).

If $\lambda^{m}=1$ for some non-negative integer $m$, there exists a non-negative integer $k$ such that $m-n_{0}=k m_{0}$, where $m_{0}$ is the number defined in the statement of the theorem. Hence there is a sequence $\left\{b_{k}\right\}_{k=0}^{\infty}$ such that $h(z)=\sum_{k=0}^{\infty} b_{k} z^{n_{0}+k m_{0}}$ for all $z$. Then

$$
\varphi_{q}(f(z))=\sum_{k=0}^{\infty} b_{k} \varphi_{p}(z)^{n_{0}+k m_{0}}=\varphi_{p}^{n_{0}}(z) g\left(\varphi_{p}^{m_{0}}(z)\right),
$$

where $g$ denotes the function analytic in $\mathbb{D}$ whose Taylor coefficients are $b_{k}$. This equation implies part (b). 
3.3. The case when $\varphi$ is a parabolic automorphism. Our last result about intertwining deals with parabolic automorphisms. In what follows, we will write $\mathbb{D}^{*}$ for the punctured unit disk $\mathbb{D} \backslash\{0\}$. Also, $\mathcal{H}\left(\mathbb{D}^{*}, \mathbb{D}\right)$ will denote the collection of all analytic functions from the punctured disk into the unit disk and $\mathcal{H}\left(\mathbb{D}^{*}, \mathbb{C}\right)$ the set of all functions which are analytic in the punctured disk.

TheOREM 6. Let $\varphi$ be a parabolic automorphism with Denjoy-Wolff point $p$, $\psi$ an arbitrary linear fractional self-map of $\mathbb{D}$, and $f$ a self-map of $\mathbb{D}$ which is conformal at the point $p$ and such that $f \circ \varphi=\psi \circ f$.

(a) If $\psi$ is the identity map, then there is a map $g \in \mathcal{H}\left(\mathbb{D}^{*}, \mathbb{D}\right)$ such that

$$
f(z)=g\left(\exp \left(-\frac{2 \pi}{\left|\varphi^{\prime \prime}(p)\right|} T_{p}(z)\right)\right), \quad z \in \mathbb{D} .
$$

The function $f$ cannot be a linear fractional map.

(b) If $\psi$ is not the identity map, denote by $q$ its Denjoy-Wolff point and write $\lambda=\frac{q \psi^{\prime \prime}(q)}{p \varphi^{\prime \prime}(p)}$. Then $\lambda \in(0,+\infty)$ and there is a map $g \in \mathcal{H}\left(\mathbb{D}^{*}, \mathbb{C}\right)$ with $g\left(\mathbb{D}^{*}\right) \subset \overline{\mathbb{H}}$ such that

$$
\left(T_{q} \circ f\right)(z)=\lambda T_{p}(z)+g\left(\exp \left(-\frac{2 \pi}{\left|\varphi^{\prime \prime}(p)\right|} T_{p}(z)\right)\right), \quad z \in \mathbb{D} .
$$

The function $f$ is a linear fractional map if and only if $g$ is constant.

Proof. We omit the proof of part (a) as its idea is essentially contained in the proof of (b) given below; just work with $\lambda=0$.

(b) By Theorem 3, we know that $\psi$ is also a parabolic automorphism. Set $g(w)=T_{q} \circ f \circ T_{p}^{-1}(w), w \in \mathbb{H}$. Clearly, $g$ is a holomorphic self-map of $\mathbb{H}$ so there exists $c=\angle \lim _{w \rightarrow \infty} \frac{g(w)}{w} \in[0,+\infty)$. Moreover, since $f$ is conformal at $p$, we have that $c>0$ and indeed (see the proof of Theorem 1) $c=\frac{q}{p} \frac{\psi^{\prime \prime}(q)}{\varphi^{\prime \prime}(p)}=\lambda$.

Wolff's Theorem implies

$$
c=\inf \left\{\frac{\operatorname{Re} g(w)}{\operatorname{Re} w}: w \in \mathbb{H}\right\}
$$

and the infimum is attained if and only if $g(w)=c w+i \beta$, for some real number $\beta$. Note that, in this case, the result follows by simply considering $g$ to be identically equal to $i \beta$. Thus, let us assume that the above infimum is not attained. In this situation, $h(w)=g(w)-\lambda w$ is a holomorphic self-map of $\mathbb{H}$. We note that the intertwining equation for $f$ transfers to an equation in $g$ in the following way: $g \circ \widehat{\varphi}=\widehat{\psi} \circ g$, where

$$
\begin{aligned}
& \widehat{\varphi}(w)=T_{p} \circ \varphi \circ T_{p}^{-1}(w)=w+i a, \\
& \widehat{\psi}(w)=T_{q} \circ \varphi \circ T_{q}^{-1}(w)=w+i b,
\end{aligned}
$$


and $a$ and $b$ are non-zero real numbers. Bearing in mind that $\lambda=b / a$, a computation shows that $h(w+i n a)=h(w)$, for all $n \in \mathbb{Z}$. In other words, $h$ is automorphic under the group $\Gamma$ generated by $\widehat{\varphi}$. Since $\widehat{\varphi}$ is parabolic, the Riemann surface $\mathbb{H} / \Gamma$ is biholomorphic to $\mathbb{D}^{*}=\mathbb{D} \backslash\{0\}$ (see [1, page 24]). Moreover, it is well-known that

$$
\pi(w)=\exp \left(-\frac{2 \pi}{|a|} w\right), \quad w \in \mathbb{H},
$$

defines a covering map $\pi$ from $\mathbb{H}$ onto $\mathbb{D}^{*}$ such that $\pi \circ \widehat{\varphi}=\pi$. Hence, we can define a holomorphic map $g$ from $\mathbb{D}^{*}$ onto $\mathbb{H}$ such that $h=g \circ \pi$. Finally, note that $|a|=\left|p \varphi^{\prime \prime}(p)\right|=\left|\varphi^{\prime \prime}(p)\right|$ so that

$$
T_{q} \circ f \circ T_{p}^{-1}(w)=\lambda w+g\left(\exp \left(-\frac{2 \pi}{\left|\varphi^{\prime \prime}(p)\right|} w\right)\right),
$$

as desired.

Trivially, if $g$ is constant, then $f$ is a linear fractional map. On the other hand, assume that $g$ is not constant and $f$ is also a linear fractional map. In this case, $\sigma=T_{q} \circ f \circ T_{p}^{-1}$ is a linear fractional map in $\mathbb{H}$ that fixes the point at $\infty$. Therefore, $\sigma(w)=d w+k$, for some $d \geq 0$ and $k \in \overline{\mathbb{H}}$. Since $g$ is not constant, we must have $d \neq \lambda$ so the function defined by $u(w)=\sigma(w)-\lambda w$ is univalent in $\mathbb{H}$. This implies that $g \circ \pi$ is univalent, which is clearly false. This ends the proof.

3.4. The intertwining equation and the sets of fixed points. We have already seen that in most cases a rigidity principle holds: a self-map $f$ of $\mathbb{D}$ which solves the intertwining equation $f \circ \varphi=\psi \circ f$ must also be a linear fractional map. However, this condition which is so often necessary, is never sufficient by itself for (5) to hold.

We now state and prove, for all possible cases, necessary and sufficient conditions for a linear fractional self-map $f$ of $\mathbb{D}$ to satisfy the intertwining equation $f \circ \varphi=\psi \circ f$ for two fixed linear fractional self-maps of the unit disk $\varphi$ and $\psi$. For this, some additional notation will be useful. Given a linear fractional self-map $h$ of $\mathbb{D}$, we denote by $\operatorname{Fix}(h)$ the collection of all fixed points of $h$ viewed as a bijective map of the Riemann sphere $\widehat{\mathbb{C}}$. That is, $\operatorname{Fix}(h)=\{w \in \widehat{\mathbb{C}}: h(w)=w\}$. We note that, whenever $h$ is not the identity, $\operatorname{Fix}(h)$ must be either a singleton or a set consisting of two points.

THEOREM 7. Let $\varphi, \psi, f$ be three linear fractional self-maps of the unit disk $\mathbb{D}$. Assume that both $\varphi$ and $\psi$ are different from the identity and denote by $p$ and $q$ their respective Denjoy-Wolff points.

(1) If $\varphi$ is elliptic, the intertwining equation $f \circ \varphi=\psi \circ f$ holds if and only if $f(\operatorname{Fix}(\varphi))=\operatorname{Fix}(\psi)$ and $\varphi^{\prime}(p)=\psi^{\prime}(q)$.

(2) If $\varphi$ is hyperbolic, the equation $f \circ \varphi=\psi \circ f$ takes place if and only if $f(\operatorname{Fix}(\varphi))=\operatorname{Fix}(\psi), f(p)=q$, and $\varphi^{\prime}(p)=\psi^{\prime}(q)$. 
(3) If $\varphi$ is parabolic, the equation $f \circ \varphi=\psi \circ f$ holds if and only if $f(\operatorname{Fix}(\varphi))=\operatorname{Fix}(\psi)$ and $f^{\prime}(p) \psi^{\prime \prime}(p)=\varphi^{\prime \prime}(p)$.

Proof. First of all, we prove that if the intertwining equation holds then always $f(\operatorname{Fix}(\varphi))=\operatorname{Fix}(\psi), f(p)=q, \varphi^{\prime}(p)=\psi^{\prime}(q)$, and, in the parabolic case, also $f^{\prime}(p) \psi^{\prime \prime}(p)=\varphi^{\prime \prime}(p)$.

An easy computation shows that $f(\operatorname{Fix}(\varphi)) \subseteq \operatorname{Fix}(\psi)$. On the one hand, if $\operatorname{Fix}(\psi)$ has two points, then $\psi$ is either elliptic or hyperbolic so, by Theorem 3 , we conclude that $\varphi$ is also elliptic or hyperbolic and, therefore, $\operatorname{Fix}(\varphi)$ also has two points. Since $f$ is univalent, we deduce that $f(\operatorname{Fix}(\varphi))$ also has two points, whence $f(\operatorname{Fix}(\varphi))=\operatorname{Fix}(\psi)$. On the other hand, if $\operatorname{Fix}(\psi)$ has just one point, and since $f(\operatorname{Fix}(\varphi))$ is non-empty, we obtain that $f(\operatorname{Fix}(\varphi))=$ $\operatorname{Fix}(\psi)$.

If $\psi$ is an elliptic automorphism, then so is $\varphi$ (see Theorem 3). Therefore, in this case, we clearly have $f(p)=q$. In the remaining cases, by Proposition 2 and the continuity of $f$ at $p$, we also conclude that $f(p)=q$.

By taking the derivatives of both sides of the intertwining equation and evaluating them at $p$, we get $f^{\prime}(p) \varphi^{\prime}(p)=\psi^{\prime}(f(p)) f^{\prime}(p)=\psi^{\prime}(q) f^{\prime}(p)$. Since $f$ is conformal at $p$, it follows that $\varphi^{\prime}(p)=\psi^{\prime}(q)$. If $\varphi$ is parabolic, then so is $\psi$ (see again Theorem 3). By differentiating the intertwining equation twice, evaluating at $p$, and using the assumption that $\varphi$ and $\psi$ are parabolic, we arrive at the formula $f^{\prime}(p) \psi^{\prime \prime}(p)=\varphi^{\prime \prime}(p)$.

We now prove the reverse implication. Suppose initially that $\varphi$ is either elliptic or hyperbolic. By assumption, $f(\operatorname{Fix}(\varphi))=\operatorname{Fix}(\psi)$. Since $f$ is injective, we find that $\operatorname{Fix}(\psi)$ has two points. In particular, $\psi$ is either elliptic or hyperbolic. If $\varphi$ is elliptic, then $f(p) \in \mathbb{D}$ and it is a fixed point of $\psi$. Thus, it follows that $f(p)=q$. If $\varphi$ is hyperbolic, it is immediate from the assumption of the theorem that $f(p)=q$.

Denote by $p_{r}$ and $q_{r}$ the remaining fixed points of $\varphi$ and $\psi$ respectively. Note that $p_{r}$ and $q_{r}$ belong to $\widehat{\mathbb{C}} \backslash \mathbb{D}$. Set $\beta(z):=z-p$ if $p_{r}=\infty$ and $\beta(z):=\frac{z-p}{z-p_{r}}$ if $p_{r} \neq \infty$ and consider the linear fractional map $H=$ $\beta \circ f^{-1} \circ \psi^{-1} \circ f \circ \varphi \circ \beta^{-1}$. One can easily check that $H(0)=0$ and $H(\infty)=\infty$. Therefore, there exists $\lambda \in \mathbb{C}, \lambda \neq 0$, such that $H(z)=\lambda z$. After a differentiation, we obtain

$$
\begin{aligned}
\lambda & =H^{\prime}(0)=\left(f^{-1} \circ \psi^{-1} \circ f \circ \varphi\right)^{\prime}(p)=\frac{(f \circ \varphi)^{\prime}(p)}{(\psi \circ f)^{\prime}\left[(\psi \circ f)^{-1} \circ(f \circ \varphi)(p)\right]} \\
& =\frac{(f \circ \varphi)^{\prime}(p)}{(\psi \circ f)^{\prime}(p)}=\frac{f^{\prime}(p) \varphi^{\prime}(p)}{\psi^{\prime}(q) f^{\prime}(p)}=\frac{\varphi^{\prime}(p)}{\psi^{\prime}(q)}=1 .
\end{aligned}
$$

Hence $\lambda=1$, meaning that $H$ is the identity. After some simple algebraic computations, we deduce that the intertwining equation holds.

Finally, we consider the remaining case when $\varphi$ is parabolic. Since $\operatorname{Fix}(\varphi)$ has just one point, it is clear that $\operatorname{Fix}(\psi)$ has a unique point and, in particular, $\psi$ is parabolic and $f(p)=q$. Now, consider the linear fractional 
maps

$$
\widehat{\varphi}=T_{p} \circ \varphi \circ T_{p}^{-1}, \quad \widehat{\psi}=T_{q} \circ \psi \circ T_{q}^{-1}, \quad F=T_{q} \circ f \circ T_{p}^{-1} .
$$

These three functions leave invariant the right-half plane and fix the point $\infty$ on the Riemann sphere. Since $\varphi$ and $\psi$ are parabolic, there exist two constants $a$ and $b$ such that $\widehat{\varphi}(w)=w+a$ and $\widehat{\psi}(w)=w+b$. Moreover, there must exist two other constants $c$ and $d$ such that $F(w)=c w+d$. Then

$$
c=\lim _{w \rightarrow \infty} \frac{F(w)}{w}=\lim _{z \rightarrow p} \frac{T_{q}(f(z))}{T_{p}(z)}=\lim _{z \rightarrow p} \frac{q+f(z)}{q-f(z)} \frac{p-z}{p+z}=\frac{q}{p} \frac{1}{f^{\prime}(p)} .
$$

From the equation $w+a=T_{p} \circ \varphi \circ T_{p}^{-1}(w)$, writing $w=T_{p}(z)$, we obtain that

$$
(p+z)(p-\varphi(z))+a(p-z)(p-\varphi(z))=(p+\varphi(z))(p-z) .
$$

By substituting $z=p$ into the second derivative of both sides of this equation, we find out that $a=p \varphi^{\prime \prime}(p)$. In a similar way, $b=q \psi^{\prime \prime}(q)$. Therefore, by assumption, we deduce that

$$
c=\frac{q}{p} \frac{1}{f^{\prime}(p)}=\frac{q}{p} \frac{\psi^{\prime \prime}(p)}{\varphi^{\prime \prime}(p)}=\frac{b}{a} .
$$

Moreover, $F(\widehat{\varphi}(w))=F(w+a)=c(w+a)+d=c w+c a+d$ and $\widehat{\psi}(F(w))=$ $F(w)+b=c w+d+b$. Since $c a=b$, we conclude that $F \circ \widehat{\varphi}=\widehat{\psi} \circ F$. After transferring this equation to the unit disk, we see that the intertwining equation $f \circ \varphi=\psi \circ f$ holds.

It is important to underline that, in the above theorem, all the hypotheses are necessary. More precisely, it is not enough to assume that $f(\operatorname{Fix}(\varphi))=$ $\operatorname{Fix}(\psi)$ in order to have the intertwining equation (compare this with the commuting case given in the beginning of Subsection 4.1 below). Some examples are clearly in order here.

ExAmple 10. For the elliptic case, choose $\varphi(z)=z / 2, \psi(z)=z / 4$, and $f=i d_{\mathbb{D}}$. Then $f(\operatorname{Fix}(\varphi))=\operatorname{Fix}(\psi)=\{0, \infty\}$ but the intertwining equation does not hold.

In the hyperbolic case, consider the functions

$$
\varphi(z)=\frac{3 z+1}{z+3}, \quad \psi_{\lambda}(z)=\frac{(\lambda+1) z+\lambda-1}{(\lambda-1) z+\lambda+1} \quad(\lambda \neq 2), \quad f=i d_{\mathbb{D}} .
$$

Then $f(\operatorname{Fix}(\varphi))=\operatorname{Fix}\left(\psi_{\lambda}\right)=\{1,-1\}$ and again the intertwining equation fails. Notice that, when $\lambda=4$, the Denjoy-Wolff point of both $\varphi$ and $\psi$ is 1 but $\varphi^{\prime}(1) \neq \psi^{\prime}(1)$. Moreover, when $\lambda=1 / 2$, the Denjoy-Wolff points of $\varphi$ and $\psi$ are 1 and -1 , respectively, and we have $\varphi^{\prime}(1)=\psi^{\prime}(-1)$.

Finally, in the parabolic case, define

$$
\varphi(z)=\frac{(2-i) z+i}{-i z+2+i}, \quad \psi(z)=\frac{(1-i) z+i}{-i z+1+i}, \quad f=i d_{\mathbb{D}} .
$$


Now we have that $f(\operatorname{Fix}(\varphi))=\operatorname{Fix}(\psi)=\{1\}$ and once again the intertwining equation does not hold.

\section{Some APplications}

We now give several applications of the results obtained in Section 2 and Section 3 or of the methods used in their proofs.

4.1. Commutation. Our first application is the description of the commutant of a linear fractional self-map of $\mathbb{D}$ other than certain exceptional automorphisms. Recall that the commutant of such $\varphi$ is the set of all holomorphic self-maps $f$ of $\mathbb{D}$ such that $f \circ \varphi=\varphi \circ f$.

It seems that until now the answer to the commutant question has been known only in a handful of cases. The following statements are classical (see [2, pp. 68-69, Theorem 4.3.5 and Theorem 4.3.6]):

- two LFT's different from the identity commute if and only if each one of them maps the set of fixed points of the other one onto itself;

- two LFT's other than the identity and with a common fixed point in the extended plane $\widehat{\mathbb{C}}$ commute if and only if they have the same set of fixed points in $\widehat{\mathbb{C}}$.

Behan [3] proved that any two commuting self-maps of $\mathbb{D}$, other than the identity or a hyperbolic automorphism, must have the same Denjoy-Wolff point. It should be noted that in the case of intertwining, even if $\varphi$ and $\psi$ have the same Denjoy-Wolff point $p$, the Denjoy-Wolff point of $f$ can be different from $p$, as our Example 8 shows (with $p=1$ being the common Denjoy-Wolff point of $\varphi$ and $\psi$ and $z=0$ being the Denjoy-Wolff point of $f)$.

An example of a function $f$, not an LFT, that commutes with an elliptic automorphism other than the identity is given by Example 9 and a description of all such maps is given in [1, Proposition 1.2.26]. The self-maps of $\mathbb{D}$ commuting with a parabolic disk automorphism $\varphi$ are also known; see [1, Proposition 1.2.27] for the statement adapted to a half-plane, and also [10]. In this case the commutant actually admits rather complicated maps, certainly not LFT's. We will show, however, that the elliptic and parabolic automorphisms $\varphi$ are the only possible exceptions.

Having proved the earlier main theorems, we now deduce the result on the commutant. The reader should note that it is not a mere corollary of our previous theorems on intertwining. Namely, one has to consider separately the elliptic and non-elliptic cases and it turns out that the elliptic case does not follow directly from the corresponding result for intertwining.

TheOREM 8. Let $\varphi$ be a linear fractional self-map of $\mathbb{D}$ which does not fall into any of the following categories: the identity, an elliptic automorphism, or a parabolic automorphism. If $f$ is a self-map of $\mathbb{D}$ different from a constant or the identity map, then $f \circ \varphi=\varphi \circ f$ holds if and only if $f$ is also 
an LFT and has the same set of fixed points in $\widehat{\mathbb{C}}$ as $\varphi$. Moreover, $f$ and $\varphi$ have the same dynamic type.

Proof. Denote by $p$ the Denjoy-Wolff point of $\varphi$. By Behan's Theorem [3], either $p$ is the Denjoy-Wolf point of $f$, or $f$ is a hyperbolic automorphism and $p$ is a fixed point of $f$. Thus, if $f$ is either parabolic or hyperbolic but not an automorphism, we have that $0<f^{\prime}(p) \leq 1$, hence $f$ is conformal at $p$. If $f$ is a hyperbolic automorphism then $f^{\prime}$ is finite, hence $f$ is again conformal. In either one of these cases, the result follows from Theorem 4.

In the elliptic case, it suffices to show that $f^{\prime}(p) \neq 0$ for the DenjoyWolff point $p$ of $\varphi$ and the statement will again follow from Theorem 4. To this end, without loss of generality we may assume that $p=0$. (Otherwise consider the conjugate application $F=\varphi_{p} \circ f \circ \varphi_{p}$ with the fixed point at the origin which also satisfies $F^{\prime}(0)=f^{\prime}(p)$.) We can, thus, start off with the assumption that $\varphi(z)=z /(c z+d)$. Note that $d \neq 0$ since $f \not \equiv$ const. Also, it follows by either Proposition 1 or Lemma 1 that $|d| \neq 1$. Now there are two possibilities.

1) $c=0$. In this case, $f(z / d)=f(z) / d$ for all $z$ in $\mathbb{D}$. This is readily seen to imply that $f(z)=a_{1} z$ for some non-zero constant $a_{1}$ (recalling that $f \not \equiv$ const and $|d| \neq 1$ ), for example, either by using the power series development or by using the following argument. Differentiation yields $f^{\prime}(z / d)=f^{\prime}(z)$; hence by induction $f^{\prime}\left(z / d^{n}\right)=f^{\prime}(z)$ for all $z \in \mathbb{D}$ so, by the uniqueness principle for analytic functions it follows that $f^{\prime} \equiv$ const.

2) $c \neq 0$. Suppose that, on the contrary, $f^{\prime}(0)=0$. Then there exists $N \geq 2$ and $a_{N} \neq 0$ such that

$$
f(z)=\sum_{n=N}^{\infty} a_{n} z^{n}=a_{N} z^{N}+o\left(z^{N}\right) .
$$

In this context, as is usual, by $o\left(z^{N}\right)$ we mean any function $g$ analytic in $\mathbb{D}$ for which $\lim _{z \rightarrow 0} g(z) / z^{N}=0$. By a standard geometric series formula,

$$
\varphi(z)=\frac{z}{c z+d}=\frac{z}{d}+o(z) .
$$

Thus, on the one hand

$$
f(\varphi(z))=a_{N} \varphi(z)^{N}+o\left(\varphi(z)^{N}\right)=\frac{a_{N}}{d^{N}} z^{N}+o\left(z^{N}\right)
$$

and, on the other hand,

$$
\varphi(f(z))=\frac{f(z)}{d}+o(f(z))=\frac{a_{N}}{d} z^{N}+o\left(z^{N}\right) .
$$

By equating the $N$-th coefficients in the last two formulas, it follows that $d=d^{N}$ which is impossible in view of the assumptions $d \neq 0,|d| \neq 1$ and $N \geq 2$. This completes the proof. 
Obviously, in the extreme case when $f$ is a constant map, it commutes with $\varphi$ if and only if $f$ is identically equal to a fixed point of $\varphi$ but since we only admit those maps $f$ for which $f(\mathbb{D}) \subset \mathbb{D}$, this can only happen in the case when $\varphi$ is elliptic.

The fact that the commutation equation $f \circ \varphi=\varphi \circ f$ implies that $f$ is an LFT whenever $\varphi$ is such, discarding the exceptional cases mentioned earlier, was also obtained in [15, Theorem 2] by using completely different methods.

4.2. Roots. The result about the commutant has an immediate application to the questions of existence of the $n$-th "roots" of a given linear fractional map, that is, of those self-maps $f$ of $\mathbb{D}$ such that $f_{n}=\varphi$. It is trivial but important to observe that the $n$-th iterate of an automorphism is trivially an automorphism. Also, an arbitrary iterate of a self-map of $\mathbb{D}$ always has the same type as the map itself.

A disk automorphism of any type (other than the identity) is easily seen to have an $n$-th root for arbitrary $n$ and this root must also be an automorphism. Indeed, a trivial argument with bijections shows that if $f$ maps $\mathbb{D}$ into itself, $f_{n}=\varphi$, and $\varphi$ is a bijection of the disk, then $f$ must also be a bijective map of $\mathbb{D}$.

The identity map is again exceptional for it can be shown to have uncountably many $n$-th roots for any $n$. One also easily checks that, given any point $p \in \mathbb{D}$, if $\lambda$ denotes any $n$-th root of unity different from 1 and $\varphi_{p}$ is defined as in (4), then every disk automorphism of the form $\varphi(z)=\varphi_{p}\left(\lambda \varphi_{p}(z)\right)$ is an $n$-th root of $i d_{\mathbb{D}}$ and $p$ is its fixed point, hence $\varphi$ is elliptic. It is easy to check that each pair $(p, \lambda)$ defines a different map, so there are indeed uncountably many of them. It is not difficult to see that all $n$-th roots of the identity map have to be elliptic automorphisms by transferring the problem to the right half-plane if necessary.

For other linear fractional self-maps $\varphi$ of $\mathbb{D}$ it turns out that a solution $f$ to the equation $f_{n}=\varphi$ exists, it must be an LFT and have the same type as $\varphi$. That is, another rigidity principle holds here. The results are as follows.

TheOREM 9. For any parabolic or hyperbolic linear fractional map $\varphi$, the equation $f_{n}=\varphi$ has a unique solution. This solution $f$ is parabolic whenever $\varphi$ is parabolic and hyperbolic whenever $\varphi$ is such.

In either case, the formula for the solution is obtained as follows: if $\tau$ denotes the Denjoy-Wolff point of $\varphi$, the map $\widehat{\varphi}=T_{\tau} \circ \varphi \circ T_{\tau}^{-1}$ becomes simply $\widehat{\varphi}(w)=A w+B$, a self-map of $\mathbb{H}$ with $A \geq 1$ and Re $B \geq 0$. Denote by $\alpha$ the only positive $n$-th root of $A$. Then

$$
f(z)=T_{\tau}^{-1}\left(g\left(T_{\tau}(z)\right)=\tau \frac{g\left(\frac{\tau+z}{\tau-z}\right)-1}{g\left(\frac{\tau+z}{\tau-z}\right)+1},\right.
$$

where

$$
g(w)=\alpha w+\frac{B}{\sum_{k=0}^{n-1} \alpha^{k}} .
$$


Proof. By virtue of the equation $f_{n}=\varphi$, it is immediate that $f$ and $\varphi$ commute:

$$
f \circ \varphi=f_{n+1}=\varphi \circ f .
$$

Hence, whenever $\varphi$ is not a parabolic automorphism, Theorem 8 shows that $f$ is also an LFT. If $\varphi$ is a parabolic automorphism, each one of its roots is again a disk automorphism, as observed earlier.

Since in all possible cases both $\varphi$ and $f$ are linear fractional transformations, the equation $f_{n}=\varphi$ becomes much easier to solve in the right halfplane $\mathbb{H}$. After applying the Cayley transform $T_{\tau}$, the equation becomes $g_{n}=\widehat{\varphi}$, where the functions $\widehat{\varphi}=T_{\tau} \circ \varphi \circ T_{\tau}^{-1}$ and $g=T_{\tau} \circ f \circ T_{\tau}^{-1}$ are selfmaps of $\mathbb{H}$. As observed earlier, $\widehat{\varphi}$ can be written in the form $\widehat{\varphi}(w)=A w+B$, with $\operatorname{Re} B \geq 0$ always and with $A=1$ in the parabolic case and $A>1$ in the hyperbolic case. Since $f$ is also an LFT with the same Denjoy-Wolff point as $\varphi$, the function $g$ will have a similar representation: $g(w)=a w+b$, $a \geq 1$ and $\operatorname{Re} b \geq 0$.

These representations allow us to find readily the formula for the $n$-th iterate $g_{n}$. After identifying the coefficients of $\widehat{\varphi}$ with those of $g_{n}$ in the equation $g_{n}=\widehat{\varphi}$, we get the system of equations

$$
a^{n}=A, \quad b\left(1+a+a^{2}+\ldots+a^{n-1}\right)=B .
$$

Writing $\alpha$ for the unique positive $n$-th root of $A$, we see that

$$
g(w)=\alpha w+\frac{B}{\sum_{k=0}^{n-1} \alpha^{k}}
$$

is one solution of the equation $g_{n}=\widehat{\varphi}$. It is actually the only one. Indeed, with any other $n$-th root of $A$ instead of the positive root $\alpha$, the map $g$ would no longer be a self-map of the right half plane.

Note also that the maps $\widehat{\varphi}$ and $g$ are of the same type, since $\alpha$ and $A$ are simultaneously equal to, or greater than, one.

The situation is radically different for elliptic maps. It can be seen that, for example, the function $\varphi(z)=-z /(3 z+4)$ has no square roots that map $\mathbb{D}$ into itself, while the automorphism $\varphi(z)=-z$ has exactly two: $f_{1}(z)=i z$ and $f_{2}(z)=-i z$. The problem is that, even though one can formally solve an equation for obtaining a linear fractional root of a given LFT, this root need not map the disk into itself. However, Proposition 1 or Lemma 1 will allow us to control effectively the number of solutions of the equation $f_{n}=\varphi$ in the case of an elliptic map different from an automorphism.

THEOREM 10. If $\varphi$ is an elliptic linear fractional self-map of $\mathbb{D}$ other than the identity and with Denjoy-Wolff point $p$, then the map $\widehat{\varphi}=\varphi_{p} \circ \varphi \circ \varphi_{p}$ has the form $\widehat{\varphi}(z)=\frac{A z}{C z+1}$ with $|A|+|C| \leq 1$ and $A \neq 1$. The number of solutions of the equation $f_{n}=\varphi$ equals the cardinality of the set

$$
\left\{a \in \mathbb{C}: a^{n}=A \text { and }|C(1-a)| \leq(1-|a|) \cdot|1-A|\right\} .
$$


Furthermore, each solution of the equation $f_{n}=\varphi$ is determined by one such root $a$ and is again an elliptic LFT of the form $f=\varphi_{p} \circ g \circ \varphi_{p}$, where

$$
g(z)=\frac{a z}{\frac{C(1-a)}{1-A} z+1} .
$$

In particular, if $\varphi$ is an elliptic automorphism other than the identity, there are exactly $n$ different solutions of the equation $f_{n}=\varphi$.

Proof. Let $\varphi$ be an elliptic linear fractional self-map of $\mathbb{D}$ with Denjoy-Wolff point $p$ in $\mathbb{D}$. Denoting by $\varphi_{p}$ the automorphism given by (4) which is an involution and interchanges $p$ and the origin, it is immediate that the map $\widehat{\varphi}=\varphi_{p} \circ \varphi \circ \varphi_{p}$ is an elliptic LFT with the Denjoy-Wolff point $z=0$. The map $\widehat{\varphi}$ in this case can be written as $\widehat{\varphi}(z)=\frac{A z}{C z+1}$ and by applying either Proposition 1 or Lemma 1, or working directly with appropriate inequalities, we readily see that it is a self-map of $\mathbb{D}$ if and only if $|A|+|C| \leq 1$.

Assuming that $\varphi$ is not an automorphism and using Theorem 8 again, we can show as before that every possible solution of the equation $g_{n}=\widehat{\varphi}$ must have the form $g(z)=\frac{a z}{c z+1}$. By matching the coefficients in the equation $g_{n}=\widehat{\varphi}$ as before, we get the system of equations

$$
a^{n}=A, \quad c\left(1+a+a^{2}+\ldots+a^{n-1}\right)=C .
$$

Note that for any $n$-th root $a$ of $A$, the sum $\sum_{j=0}^{n-1} a^{j} \neq 0$. Otherwise we would have $0=1-a^{n}=1-A$ and $C=0$, meaning that $\widehat{\varphi}$ and therefore also $\varphi$ is the identity map, the case excluded from the start. Thus, we can solve the system of equations (7) and infer that the functions of the form

$$
g(z)=\frac{a z}{\frac{C}{\sum_{j=0}^{n-1} a^{j}} z+1}=\frac{a z}{\frac{C(1-a)}{1-A} z+1},
$$

where $a$ denotes any one of the $n$-th roots of $A$, are the only possible solutions of the equation $g_{n}=\widehat{\varphi}$. Reasoning as before, such a map $g$ will be a self-map of $\mathbb{D}$ if and only if

$$
|C(1-a)| \leq(1-|a|) \cdot|1-A| .
$$

Therefore, the solutions of the equation $g_{n}=\widehat{\varphi}$, if any, will be only those maps $g$ as above for which inequality (8) is verified. This easily leads to the formula for the solutions of the equation $f_{n}=\varphi$ given in the statement of the theorem.

Now let $\varphi$ be an elliptic automorphism. The map $\widehat{\varphi}=\varphi_{p} \circ \varphi \circ \varphi_{p}$ is an automorphism that fixes the origin, hence a rotation: $\widehat{\varphi}(z)=\lambda z, \lambda \in$ $\partial \mathbb{D} \backslash\{1\}$. The map $f$ (and therefore also $g$ ) is a disk automorphism other than the identity. Since $g$ and $\widehat{\varphi}$ commute and $\widehat{\varphi}$ fixes the origin, the same must be true of $g$, hence it is also a rotation. Thus, if $\mu$ is any $n$-th root of $\lambda$, it is immediate that the rotation $g(z)=\mu z$ is a solution of the equation $g_{n}=\widehat{\varphi}$ and there can be no other solutions. 
4.3. A remark on the Koenigs embedding problem for semigroups. We finally address some aspects of the so-called Koenigs embedding problem for semigroups of analytic functions. Recall that an indexed family $\mathcal{G}=$ $\left\{g_{t}: t \in[0, \infty)\right\}$ of analytic self-maps of $\mathbb{D}$ is said to be a semigroup if it is closed and additive under composition: $g_{s} \circ g_{t}=g_{s+t}$ for all $s, t \in[0, \infty)$ and the function $t \mapsto g_{t}$ is strongly continuous, meaning that $g_{s} \rightarrow g_{t}$ uniformly on the compact sets in $\mathbb{D}$ as $s \rightarrow t$. Note that the additivity condition implies that $g_{0}$ is the identity mapping. Much about semi-groups of analytic functions can be found in the survey article [26] and in the monograph [25].

In relation to the elliptic case, it is clear that there are semigroups of automorphisms in which the identity equals $g_{t}$ for more than one value of $t$ (for example, when $g_{t / 2}$ coincides with some automorphism $\varphi_{p}$, an involution).

The following is a natural and fundamental question about semi-groups of analytic self-maps of the disk: when does such a semi-group contain a linear fractional map? The answer has recently been given in [8, Theorem 3.2]: this happens if and only if each member of the semi-group $\mathcal{G}=\left\{g_{t}: t \in[0, \infty)\right\}$ is a linear fractional map. Moreover, all of its members $g_{t}$ with $t \neq 0$ are linear fractional maps of the same type as the given LFT. This can be seen as follows from our findings here. Suppose that $\mathcal{G}$ contains an LFT (not the identity), say $\varphi=g_{t}$, for some $t>0$. As long as $\varphi$ is not an elliptic or parabolic automorphism, the commutation relation

$$
g_{s} \circ g_{t}=g_{s+t}=g_{t} \circ g_{s}
$$

and Theorem 8 will imply that any other member $g_{s}$ of $\mathcal{G}$ must also be an LFT and of the same type as $\varphi$. If $\varphi$ is an automorphism, the conclusion follows in a different but simple manner and is well known.

The corollary below provides further details about embedding an LFT into a semigroup. The first part of the statement (on the uniqueness of the semigroup that contains a given LFT) must be known to the experts in various cases and may have simpler proofs as well. We state it here because it does not seem easy to give an explicit reference that would cover all the cases, and also because of the novelty of the method of proof. The main idea here is to recover the unique semigroup from just one of its elements by extracting successive square roots, thus relying on our Theorem 9 and Theorem 10. The same idea appears in the second part, which refers to the elliptic case. Our method also yields a criterion somewhat different from the analytic condition presented in [15], [16], and [25, Proposition 5.9.5], as well as from the geometric criterion given most recently in [8, Proposition 3.4].

COROLlaRY 1. The following assertions hold for semigroups of self-maps of the unit disk.

(i) Given a parabolic or hyperbolic linear fractional self-map $\varphi$ of $\mathbb{D}$ and a positive number $s$, there is a unique semigroup $\mathcal{G}=\left\{g_{t}: t \in\right.$ $[0, \infty)\}$ of self-maps of $\mathbb{D}$ such that $g_{s}=\varphi$. Every elliptic disk automorphism is a member of some semigroup (not necessarily unique). 
(ii) Every elliptic, non-automorphic, linear fractional self-map $\varphi$ of $\mathbb{D}$ is conjugate to a map of the form $(A z) /(C z+1)$, where $|A|+|C| \leq$ 1, as was pointed out in Theorem 10. Such a map $\varphi$ belongs to some semigroup of self-maps of the disk if and only if there exists a sequence $\left\{a_{n}\right\}_{n=1}^{\infty}$ such that $a_{0}=A$, every $a_{n}(n \geq 1)$ is one of the square roots of the number $a_{n-1}$, and all $a_{n}$ belong to the nontangential approach (Stolz type) region

$$
S=\left\{z \in \mathbb{D}: \frac{|C|}{|1-A|}|1-z| \leq 1-|z|\right\}
$$

with vertex at $z=1$.

Proof. In order to prove assertion (i) of the theorem, let us assume that $\varphi$ is either hyperbolic or parabolic. The function $\varphi$ will always have a square root which is an LFT of the same type as $\varphi$. In view of the rule $g_{t / 2} \circ g_{t / 2}=g_{t}=\varphi$, this root must be equal to $g_{t / 2}$. Since the root is again a map of the same type as $\varphi$, it will have a unique root itself, and this root is again an LFT of the same type as $\varphi$; we may proceed inductively to conclude that every member of $\mathcal{G}$ of the form $g_{t / 2^{n}}$ is determined uniquely by $\varphi$. By composition, $g_{m t / 2^{n}}$ is also determined in a unique fashion for all non-negative integers $m, n$. The dyadic rational numbers $m / 2^{n}$ are dense in $[0, \infty)$, so by strong continuity every member of the semigroup $\mathcal{G}$ is determined uniquely by the initial map $\varphi$.

The statement for elliptic automorphisms can be proved in an analogous way, except for the uniqueness part: $\varphi$ may have more than one root but exactly one of these roots must equal $g_{t / 2}$, so pick that one and proceed as before to show that all maps $g_{m t / 2^{n}}$ are elliptic automorphisms. Finally, a standard arguments involving Hurwitz's theorem shows that any locally uniform limit of such maps is also univalent or a constant map. Since a semigroup cannot contain constant maps, it follows that the limit function is univalent; it follows rather easily that it must actually be an elliptic automorphism.

Let us now prove (ii). If an elliptic non-automorphic map $\varphi$ belongs to a semigroup $\mathcal{G}$, say $\varphi=g_{t}$ with $t>0$, then $g_{t / 2}$ must be one of the possible square roots of $\varphi$ as described in Theorem 10. But we have seen that the only possible square roots of the elliptic non-automorphic function $(A z) /(C z+1)$ have the form

$$
\frac{a z}{\frac{C(1-a)}{1-A} z+1}, \quad a^{2}=A
$$

where $|C(1-a)| \leq(1-|a|)|1-A|$. An easy computation shows that every possible square root of this root must have the form

$$
\frac{b z}{\frac{C(1-b)}{1-A} z+1}, \quad b^{2}=a,
$$

and is a self-map of $\mathbb{D}$ if and only if $|C(1-b)| \leq(1-|b|)|1-A|$. Thus, both the form of the map and the condition for being a self-map are completely 
analogous to the previous inequality, the number $a$ now being replaced by its square root $b$. This allows us to conclude inductively that the sequence $\left\{a_{n}\right\}_{n=1}^{\infty}$ defined by $a_{0}=A$ and $a_{n}$ being equal to one of the square roots of the number $a_{n-1}, n \geq 1$, has the required property: all numbers $a_{n}$ belong to the region

$$
S=\left\{z \in \mathbb{D}: \frac{|C|}{|1-A|}|1-z| \leq 1-|z|\right\} .
$$

It should be pointed out that when $C \neq 0$ we can never get the identity map in this process of root extractions. In the case $C=0$, the region $S$ degenerates into the entire unit disk, so the condition of belonging to it is trivially fulfilled and the roots of $A z$ are easily found to be $a z$, where $a^{n}=A$.

Conversely, if there exists a sequence $\left\{a_{n}\right\}_{n=1}^{\infty}$ as above, its every member $a_{n}$ will satisfy condition (8) for the existence of the root of order $2^{n}$ of the initial map $\varphi$, which allows us to proceed in the same way as in the other cases and determine the unique semigroup $\mathcal{G}$ to which $\varphi$ belongs by determining all members $g_{t / 2^{n}}$ first.

Note that in the above condition the sequence $\left\{\left(1-\left|a_{n}\right|\right) /\left|1-a_{n}\right|\right\}_{n=0}^{\infty}$ is decreasing by the definition of $a_{n}$ and the elementary inequality

$$
\frac{1-\left|a_{n}\right|}{\left|1-a_{n}\right|}=\frac{1-\left|a_{n+1}\right|^{2}}{\left|1-a_{n+1}^{2}\right|} \geq \frac{1-\left|a_{n+1}\right|}{\left|1-a_{n+1}\right|} \text {. }
$$

\section{REFERENCES}

[1] M. Abate, Iteration Theory of Holomorphic Maps on Taut Manifolds, Mediterranean Press, Commenda di Rende 1989.

[2] A. Beardon, The Geometry of Discrete Groups, Graduate Texts in Mathematics 91, Springer-Verlag, New York - Heidelberg - Berlin 1983.

[3] D. F. Behan, Commuting analytic functions without fixed points, Proc. Amer. Math. Soc. 37 (1973), no. 1, 114-120.

[4] C. Bisi and G. Gentili, Commuting holomorphic maps and linear fractional models, Complex Variables Theory Appl. 45 (2001), no. 1, 47-71.

[5] P. S. Bourdon, E. E. Fry, C. Hammond, and C. H. Spofford, Norms of linear-fractional composition operators, Trans. Amer. Math. Soc. 356 (2004), no. 6, 2459-2480.

[6] D. Burns and S. G. Krantz, Rigidity of holomorphic mappings and a new Schwarz lemma at the boundary, J. Amer. Math. Soc. 7 (1994), no. 3, 661-676.

[7] F. Bracci, Fixed points of commuting holomorphic mappings other than the Wolff point, Trans. Amer. Math. Soc. 355 (2003), no. 6, 2569-2584.

[8] F. Bracci, M. D. Contreras, and S. Díaz-Madrigal, Infinitesimal generators associated with semigroups of linear fractional maps, J. Anal. Math. 102 (2007), 119-142.

[9] F. Bracci, R. Tauraso, and F. Vlacci, Identity principles for commuting holomorphic self-maps of the unit disc, J. Math. Anal. Appl. 270 (2002), no. 2, 451-473.

[10] M. D. Contreras, S. Díaz-Madrigal, and Ch. Pommerenke, Some remarks on the Abel equation in the unit disk, J. London Math. Soc. 75 (2007), 623-634.

[11] M. D. Contreras, S. Díaz-Madrigal, and Ch. Pommerenke, Iteration in the unit disk: the parabolic zoo. To appear in: Proceedings of the Conference "Complex and Harmonic Analysis" held in Thessaloniki, Greece in May of 2006, Destech Publications Inc. Editors: A. Carbery, P. L. Duren, D. Khavinson, and A. G. Siskakis. 
[12] C. Cowen, Iteration and the solution of functional equations for functions analytic in the unit disk, Trans. Amer. Math. Soc. 265 (1981), no. 1, 69-95.

[13] C. Cowen, Commuting analytic functions, Trans. Amer. Math. Soc. 283 (1984), no. 2, 685-695.

[14] C. Cowen and B. MacCluer, Composition Operators on Spaces of Analytic Functions, Studies in Advanced Mathematics, CRC Press, Boca Raton 1995.

[15] F. Jacobzon, S. Reich, and D. Shoikhet, Linear fractional mappings: invariant sets, semigroups, and commutativity, J. Fixed Point Theory Appl. 5 (2009), no. 1, 63-91.

[16] V. Khatskevich, S. Reich, and D. Shoikhet, Abel-Schröder equations for linear fractional mappings and the Koenigs embedding problem, Acta Sci. Math. (Szeged) 69 (2003), no. 1-2, 67-98.

[17] M. J. Martín, Composition operators with linear fractional symbols and their adjoints, First Advanced Course in Operator Theory and Complex Analysis (Seville 2004), pp. 105-112 (A. Montes-Rodríguez, editor), University of Seville 2006.

[18] P. Poggi-Corradini, On the uniqueness of classical semiconjugations for self-maps of the disk, Comput. Methods Funct. Theory 6 (2006), no. 2, 403-421.

[19] Ch. Pommerenke, Polymorphic functions for groups of divergence type, Math. Ann. 258 (1982), 353-366.

[20] Ch. Pommerenke, Boundary Behaviour of Conformal Maps, Springer-Verlag, Berlin 1992.

[21] W. Rudin, Real and Complex Analysis, Third Edition, McGraw-Hill, New York, St. Louis, etc. 1987.

[22] J. H. Shapiro, Composition Operators and Classical Function Theory, SpringerVerlag, New York 1993.

[23] J. H. Shapiro, W. Smith, and D. Stegenga, Geometric models and compactness of composition operators, J. Funct. Anal. 127 (1995), no. 1, 21-62.

[24] A. L. Shields, On fixed points of commuting analytic functions, Proc. Amer. Math. Soc. 15 (1964), 703-706.

[25] D. Shoikhet, Semigroups in geometrical function theory, Kluwer Academic Publishers, Dordrecht 2001.

[26] A. G. Siskakis, Semigroups of composition operators on spaces of analytic functions, a review. In Studies on composition operators (Laramie, WY, 1996), 229-252, Contemp. Math. 213, Amer. Math. Soc., Providence, RI 1998.

[27] G. Valiron, Sur l'itération des fonctions holomorphes dans un demi-plan, Bulletin Sc. math. 55 (1931), 105-128.

[28] F. Vlacci, On commuting holomorphic maps in the unit disc of $\mathbb{C}$, Complex Variables Theory Appl. 30 (1996), no. 4, 301-313. 
Departamento de Matemática Aplicada II, Escuela Técnica Superior de Ingenieros, Universidad de Sevilla, Camino de los Descubrimientos s/n, 41092 SEVILle, SPAin

E-mail address: contreras@us.es

$U R L:$ http://personal.us.es/contreras

Departamento de Matemática Aplicada II, Escuela Técnica Superior de Ingenieros, Universidad de Sevilla, Camino de los Descubrimientos s/n, 41092 SEVIlle, Spain

E-mail address: madrigal@us.es

Departamento de Matemáticas, Módulo 17, Edificio de Ciencias, UniversiDad Autónoma de Madrid, 28049 Madrid, Spain

E-mail address: mariaj.martin@uam.es

Departamento de Matemáticas, Módulo 17, Edificio de Ciencias, UniversiDAD Autónoma de MAdrid, 28049 MAdrid, SpAin

E-mail address: dragan.vukotic@uam.es

$U R L:$ http://www.uam.es/dragan.vukotic 